\title{
Stubborn and Dead-Zone Redesign for Nonlinear Observers and Filters
}

\author{
Daniele Astolfi, Angelo Alessandri, and Luca Zaccarian
}

\begin{abstract}
We propose a redesign paradigm for stable estimators by introducing a saturation or a dead-zone nonlinearity with adaptive thresholds on the output injection term. Such nonlinearities allow improving the sensitivity to measurement noise in different scenarios (impulsive disturbances or persistent noise such as sensor bias), while preserving the asymptotic convergence properties of the original observer. These redesigns apply to a broad class of state estimators, including linear observers, observers for input-affine systems, observers for Lipschitz systems, observers based on the circle criterion, high-gain observers, standard and extended Kalman filters. Simulation results confirm the effectiveness of both the stubborn and dead-zone redesigns.
\end{abstract}

Index Terms-Nonlinear observers, saturation, dead-zone, input-to-state stability

\section{INTRODUCTION}

State observers are the basic instrument to detect abnormal operating conditions such as faults or malfunctions and feed controllers when not all the state variables are accessible. While state estimation for linear systems is a well studied topic, beginning with the seminal papers by Kalman [31] and Luenberger [36], many different approaches for nonlinear systems are still under development. See, among others, [4], [11], [14], [15], [19], [28], [33], [39]. However, most of these approaches focus on the convergence properties of the estimation error in nominal conditions, that is, when the plant's model is known perfectly and when no measurement noise is affecting the outputs. Indeed, at present, few tools are available to analyze the effect of the measurement noise on the estimation error in a nonlinear context. Most of the existing works follow a "worst-case approach" such as $H_{\infty}$ gains, Lyapunov bounds or input-to-state stability gains. An attempt to address high-frequency measurement noise was recently investigated for high-gain observers [12].

Depending on the characteristics of the measurement noise, different approaches may be pursued to improve the observer performances. For instance, in the case of output measurements affected by outliers, that is, perturbations of impulsive nature affecting the measurement for a very short time, the majority of the existing methods focus on a discrete-time representation and mainly deal with identification problems, see, e.g., [2], [25], [44] and the references therein. When considering high-frequency measurement noise, a number of high-gain approaches have been developed, see, e.g., [1], [10], [11], [13], [18], [20], [42], [43]. These techniques, however, strongly exploit the particular structure of the observer at hand and can be difficult to extend to other approaches. To the best of the

D. Astolfi is with Univ Lyon, Université Claude Bernard Lyon 1, CNRS, LAGEP UMR 5007, 43 Boulevard du 11 Novembre 1918, F-69100, Villeurbanne, France (daniele.astolfi@univ-lyon1.fr).

A. Alessandri is with the University of Genova (DIME), Italy, (alessandri@dime.unige.it).

L. Zaccarian is with LAAS-CNRS, Universite de Toulouse, CNRS, Toulouse, France, and with Dept. of Industrial Engineering, University of Trento, Trento, Italy (zaccarian@laas.fr).

Research supported in part by ANR via grant HANDY, number ANR18-CE40-0010. authors' knowledge, a general methodology to improve the sensitivity to measurement noise applicable to a broad class of estimators is still missing.

The objective of this work is that of proposing a systematic procedure to redesign a given observer or filter in order to improve its performances in noisy scenarios. We suppose that the estimator has already been designed and it satisfies some mild, possibly local, input-to-state stability (ISS) properties [39], [40]. For instance, any of the techniques proposed in [3], [4], [7], [8], [10], [11], [13][15], [17]-[19], [27]-[29], [33], [34], [36], [37], [39], [43] enjoy these properties. Then, we propose two different methodologies to redesign the output injection term, both of them preserving ISS. The first one, called stubborn redesign, was first introduced in the context of linear systems [5], [6], and high-gain observers [9], and aftewards used in Kalman filters [26], neural networks [35] and synchronization [21], [22]. It consists in adding an adaptive saturation to the output injection error in the observer dynamics so as to reduce the sensitivity to measurement outliers. The second one, called deadzone redesign, generalizes the works [23], [24] for Luenberger and high-gain observers, where a "dead-zonated" output injection with a dynamically-adapted dead band enables mitigating the effect of bounded and persistent measurement noise. The two designs can be also combined. The novelty of these approaches is that the saturation/dead-zone levels are not fixed but they are dynamically adjusted to obtain desirable properties. For instance, the stubborn redesign well addresses the presence of sporadic measurement outliers. The saturation threshold regulates the trimming action on the output injection term by shrinking it to zero, thereby making the observer increasingly "stubborn" about its current estimate [6], [9]. In this way, possible outliers do not directly reach the error dynamics because they are mitigated by the limiting effect of the saturation. On the other hand, persistent estimation errors gradually cause an increase of the saturation threshold and become increasingly important in the error dynamics, so as to guarantee ISS for the estimation error dynamics. The same analysis is accomplished for the dead-zone redesign, i.e., by considering estimators with a dead-zonated output injection, well suited to improving the rejection of persistent bounded measurement noise [24].

As compared to [5], [6], [9], [23], [24], this work extends our previous results by taking into account a more general class of nonlinear plants (which can also be multi-output) and a broader range of nonlinear estimators including, among others, the extended Kalman filter (EKF). Furthermore, the dynamics of the saturation/dead-zone level scales linearly with the output error, instead of quadratically as in [5], [6], [23], [24]. We also adopt a new setup by using separate thresholds for the different output channels, which provides improved responses.

The paper is organized as follows. In Section II, we describe the system and estimator models we deal with. The main results under general sufficient conditions for the stubborn and dead-zone redesigns are presented in Sections III and IV, respectively. Estimators based on combining the two redesign paradigms are given in Section V. Section 
VI illustrates our redesigns for a number of well-known observer classes, i.e., linear Luenberger observers, observers for Lipschitz nonlinear systems [37], [45], obsevers for input-affine systems [17], [27], [29], EKFs [19], [34], [38], and low-power high-gain observers [11], and is completed by a brief discussion on its application to other nonlinear state-estimators (such as those in [4], [8], [14], [16], [18], [28], [33], [40]). Finally, the results of the proposed redesigns are applied in a simulation case study with the EKF in Section VII. Conclusions are drawn in Section VIII.

Notation. We define $\mathbb{R}_{\geq 0}:=[0, \infty)$. Given $x \in \mathbb{R}^{n}$ and $y \in \mathbb{R}^{m}$, $(x, y)$ denotes $\left[x^{\top} y^{\top}\right]^{\top}$. Given $x \in \mathbb{R}^{n},|x|:=|x|_{2}$ is its Euclidean norm, $\left\{\left.x\right|_{1}:=\sum_{i}\left|x_{i}\right|\right.$ is its Taxicab (or Manhattan) norm, and $|x|_{\mathrm{cw}}:=\left(\left|x_{1}\right|, \ldots,\left|x_{n}\right|\right)$, satisfying

$$
\begin{aligned}
|x|_{2} \leq|x|_{1} & \leq \sqrt{n}|x|_{2} & & \forall x \in \mathbb{R}^{n} \\
\left.|| x\right|_{\text {cw }}-|y|_{\text {cw }} \mid & \leq \sqrt{n}|x-y| & & \forall x, y \in \mathbb{R}^{n} .
\end{aligned}
$$

$|A|$ denotes the standard induced matrix norm of the real matrix $A, I$ denotes the identity matrix, and $\operatorname{diag}\left(a_{1}, \ldots, a_{n}\right)$ is a diagonal matrix having diagonal entries $a_{1}, \ldots, a_{n}$. If $A$ is symmetric, $\bar{\lambda}_{A}$ and $\underline{\lambda}_{A}$ denote its maximum and minimum eigenvalues, respectively; in addition, $A<0(A>0)$ means that $A$ is negative (positive) definite and $A \leq 0(A \geq 0)$ means that $A$ is negative (positive) semidefinite. We denote with $\operatorname{vec}(A) \in \mathbb{R}^{n m}$ the vectorization of a matrix $A \in \mathbb{R}^{n \times m}$, namely $\operatorname{vec}(A):=$ $\left(a_{11}, \ldots, a_{1 m}, a_{21}, \ldots, a_{2 m}, \ldots, a_{n 1}, \ldots, a_{n m}\right)$, where $a_{i j}$ is the $i, j$ entry of $A$. If $A=A^{\top}$, then $\operatorname{vec}(A) \in \mathbb{R}^{n(n+1) / 2}$ lists symmetric elements only once. Given $\delta>0$, we define $\mathbb{B}_{\delta}:=\left\{x \in \mathbb{R}^{n}\right.$ : $|x| \leq \delta\}$. For a time-varying signal $x: \mathbb{R}_{\geq 0} \rightarrow \mathbb{R}^{n}$, let $\|x\|_{\infty}:=$ $\sup _{t \in[0, \infty)}|x(t)|$. Given $x \in \mathbb{R}, \sigma \in \mathbb{R}_{\geq 0}$, define $\operatorname{sat}_{\sigma}(x):=$ $\max \{-\sigma, \min \{\sigma, x\}\}$. Given $x \in \mathbb{R}^{n}, \sigma:=\left(\sigma_{1}, \ldots, \sigma_{n}\right) \in \mathbb{R}_{\geq 0}^{n}$, define $\operatorname{sat}_{\sigma}(x):=\left(\operatorname{sat}_{\sigma_{1}}\left(x_{1}\right), \ldots, \operatorname{sat}_{\sigma_{n}}\left(x_{n}\right)\right)$ and $\mathrm{dz}_{\sigma}(x):=$ $x-\operatorname{sat}_{\sigma}(x)$. We refer to [41] for standard definitions of class $\mathcal{K}, \mathcal{K}_{\infty}$ and $\mathcal{K} \mathcal{L}$ functions. For a locally Lipschitz function $V(t)$, we define the (upper) Dini derivative of $V$ at $t$ as $D^{+} V(t):=$ $\lim \sup _{h \rightarrow 0^{+}}(V(t+h)-V(t)) / h$.

\section{SYSTEM AND OBSERVER DESCRIPTION}

\section{A. Problem Statement}

In this work we consider nonlinear systems of the form

$$
\dot{x}=f(x, u)+w, \quad y=h(x)+v,
$$

where $x \in \mathbb{R}^{n}$ is the state, $u \in \mathbb{R}^{p}$ is a known input, $y \in \mathbb{R}^{m}$ is the measured output, $w \in \mathbb{R}^{n}$ is some external disturbance, and $v \in \mathbb{R}^{m}$ represents the sensor measurement noise. For system (3) we suppose to know an observer providing an asymptotic estimate $\hat{x}$ of state $x$. A fairly general expression including, among others, Luenberger observers, Kalman filters, observers for input-affine systems, observers for Lipschitz systems, observer based on the circle criterion, high-gain observers, and low-power high-gain observers, [4], [8], [11], [14], [14], [17]-[19], [27]-[29], [33], [34], [37], [38], [40], [45] (see the details in Section VI), corresponds to

$$
\dot{z}=\varphi(z, u)+G \kappa(z, y-h(\hat{x})), \quad \hat{x}=\psi(z)
$$

where $z \in \mathcal{Z} \subseteq \mathbb{R}^{\varrho}$, with $\varrho$ integer such that $\varrho \geq n$, is the state of the observer, and $\hat{x} \in \mathbb{R}^{n}$ is the estimate of $x$. The functions $\varphi: \mathbb{R}^{\varrho} \times \mathbb{R}^{p} \rightarrow \mathbb{R}^{\varrho}, \kappa: \mathbb{R}^{\varrho} \times \mathbb{R}^{m} \rightarrow \mathbb{R}^{\rho}$, and $\psi: \mathbb{R}^{\varrho} \rightarrow \mathbb{R}^{n}$ are locally Lipschitz and $G$ is a matrix of dimension $\varrho \times \rho$. Function $\kappa$ denotes the output injection term and is such that $\kappa(z, 0)=0$ for all $z \in \mathbb{R}^{\varrho}$, which ensures that the origin is an equilibrium point for the error dynamics in the absence of disturbances. Without loss of generality, we could consider functions $\kappa$ that also depend on the input $u$. This is not explicitly written in the following as it would unnecessarily complicate the notation. Matrix $G$, satisfying $|G| \leq 1$ without loss of generality, is a selection matrix encompassing the fact that the output injection term $\kappa$ might affect only part of the $z$ dynamics (as for the observers of Sections VI-C and VI-D).

We further suppose that observer (4) has already been designed in order to satisfy certain input-to-state stability (ISS) properties. Our objective is then to redesign (4) to improve its performances in the presence of certain types of sensor measurement noise $v$. Two redesign approaches are proposed in this paper. The first one, based on the preliminary ideas of [6], [9], uses a saturation nonlinearity and turns out to be effective when the noise $v$ is of impulsive nature, i.e., whenever outliers degrade the measurement $y$. The second one, originally presented in [23], [24], is based on a dead-zone nonlinearity and mitigates the effect of bounded persistent sensor noises such as a sensor bias. With a unifying point of view, we will show that an ISS property of observer (4) allows applying any of the two approaches. Furthermore, for each redesign strategy, ISS also holds for the redesigned observer, thus allowing for a mixed (combined) redesign. A more detailed performance analysis will be given in Sections III-B and IV-B for the case when both (3) and (4) are linear, to further certify the usefulness of the two proposed techniques.

\section{B. Main Assumptions}

To characterize the properties of observer (4) in terms of domain of attraction (local, semi-global or global) and ISS w.r.t. disturbances, we first suppose that the state $x$ of system (3) and signals $u, v, w$ evolve in some given sets, which may be compact or unbounded according to the selected class of systems and observers. Specific classes of observers are addressed in detail in Section VI.

Assumption 1 There exist $\mathcal{X}_{0} \subseteq \mathcal{X} \subseteq \mathbb{R}^{n}, \mathcal{U} \subseteq \mathbb{R}^{p}$, and a compact set $\mathcal{W} \subset \mathbb{R}^{n}$ such that the trajectories of (3), with initial conditions in $\mathcal{X}_{0}$, input $u(t) \in \mathcal{U}$, and disturbances $w(t) \in \mathcal{W}$ for all $t \geq 0$, remain in $\mathcal{X}$ for all $t \geq 0$.

Our redesign approach is based on conditioning the output injection term in (4) by way of suitable nonlinearities comprising saturations and deadzones. These redesign methods essentially perturb the output injection term $\kappa$ in (4) in ways that are well represented by the following version of observer (4) with perturbed injection

$$
\dot{z}=\varphi(z, u)+G \kappa(z, y-h(\hat{x}))+G d, \quad \hat{x}=\psi(z)
$$

where $d \in \mathbb{R}^{\rho}$ is a generic disturbance affecting the observer dynamics in directions matching the input channel of $\kappa$ (that is, through the same matrix $G$ ). Due to the generality of our context, where $z$ may be more than a mere copy of the plant dynamics, we emphasize that $w$ in (3) and $d$ in (5) may act differently on the estimation error dynamics. As an example, this is true in the case study of Section VI-E.

In the rest of this paper we will ask observer (4) to be ISS w.r.t. the disturbances $w, v$ affecting the plant (3) and w.r.t. the disturbance $d$ acting on the observer (5). The desired ISS characterization is given in terms of an ISS-Lyapunov function, and we will say that observer (4) is an ISS observer if Property 1 below is satisfied. It turns out that, for most classes of observers, ISS w.r.t. $d$ is obtained for free once ISS w.r.t. $w$ and $v$ is guaranteed (although the ISS-gains may be different). For further details, see Section VI.

Property 1 Observer (4) is an ISS observer for system (3) on $\mathcal{Z} \subseteq$ $\mathbb{R}^{\varrho}$ if there exist a locally Lipschitz function $V: \mathcal{X} \times \mathcal{Z} \rightarrow \mathbb{R}_{\geq 0}$ satisfying $V(x, z) \geq 0$ for all $(x, z) \in \mathcal{X}, \mathcal{Z}$, functions $\underline{\alpha}, \bar{\alpha} \in \mathcal{K}_{\infty}^{-}$, 
a right inverse $\psi^{-R}$ of function $\psi$ (namely a function satisfying $x=\psi\left(\psi^{-R}(x)\right)$, for all $\left.x \in \mathcal{X}\right)$, compact sets $\mathcal{V} \subset \mathbb{R}^{m}, \mathcal{D} \subset \mathbb{R}^{\rho}$, and constants $\bar{\kappa}, c, c_{v}, c_{w}, c_{d}>0$ such that the following inequalities hold

$$
|G| \leq 1, \quad\left|\kappa\left(z, y_{1}\right)-\kappa\left(z, y_{2}\right)\right| \leq \bar{\kappa}\left|y_{1}-y_{2}\right|
$$

for all $z \in \mathcal{Z}$ and $y_{1}, y_{2} \in \mathbb{R}^{m}$,

$$
\underline{\alpha}(|x-\psi(z)|) \leq V(x, z) \leq \bar{\alpha}\left(\left|\psi^{-R}(x)-z\right|\right)
$$

for all $(x, z) \in \mathcal{X} \times \mathcal{Z}$,

$$
D^{+} V \leq-c V(x, z)+c_{v}|v|+c_{w}|w|+c_{d}|d|
$$

for all $(x, z) \in \mathcal{X} \times \mathcal{Z}, u \in \mathcal{U}$ and all $(v, w, d) \in \mathcal{V} \times \mathcal{W} \times \mathcal{D}$, along the dynamics of system (3) interconnected with the perturbedinjection observer (5).

Condition (6) of Property 1 states that function $\kappa(z, \cdot)$ is globally Lipschitz uniformly in $z \in \mathcal{Z}$. Conditions (7) and (8) state that (5) is an asymptotic observer for system (3) and that the estimation error $|x-\hat{x}|$ is input-to-state stable ${ }^{1}$ w.r.t. the measurement noise $v$ and disturbances $w, d$, namely there exists $\beta \in \mathcal{K} \mathcal{L}, \vartheta \in \mathcal{K}_{\infty}$ such that all solutions $t \mapsto(x(t), z(t))$ belonging to $\mathcal{X} \times \mathcal{Z}$ for all $t \geq 0$ satisfy

$$
\begin{aligned}
|\hat{x}(t)-x(t)| \leq \beta\left(\mid \psi^{-R}(\right. & x(0))-z(0) \mid, t) \\
& +\vartheta\left(\|v\|_{\infty}+\|w\|_{\infty}+\|d\|_{\infty}\right) .
\end{aligned}
$$

Motivated by [39], the above stated ISS property is coordinatedependent when $\mathcal{X}$ and $\mathcal{U}$ are unbounded (as in Section VI-B for example). It is thus emphasized that guaranteeing Property 1 may require selecting a clever set of coordinates. Next, we suppose that the ISS-Lyapunov function $V$ of Property 1 satisfies an output-growth condition.

Property 2 There exist constants $\ell_{0}, \ell_{1}, \ell_{v}, \ell_{w}, \ell_{d}>0$ such that the following holds for system (3), observer (5), and function $V$ of Property 1 with $\hat{x}=\psi(z)$,

$$
\begin{gathered}
|h(x)-h(\hat{x})| \leq \ell_{0} V(x, z) \\
\left|D^{+}(h(x)-h(\hat{x}))\right| \leq \ell_{1} V(x, z)+\ell_{v}|v|+\ell_{w}|w|+\ell_{d}|d|
\end{gathered}
$$

for all $(x, z) \in \mathcal{X} \times \mathcal{Z}, u \in \mathcal{U}$ and all $(v, w, d) \in \mathcal{V} \times \mathcal{W} \times \mathcal{D}$.

Condition (9) requires that $V$, which is an ISS-Lyapunov function for the estimation error $|x-\hat{x}|$, has the same growth as the output error function $|h(x)-h(\hat{x})|$. In the forthcoming Section VI, we show that a number of existing observers enjoy Properties 1, 2 and discuss how to select functions $V, \underline{\alpha}, \bar{\alpha}, \psi^{-R}$ and constants $\bar{\kappa}, c, c_{v}, c_{w}, c_{d}, \ell_{0}, \ell_{1}, \ell_{v}, \ell_{w}, \ell_{d}$ for such observers.

\section{Dynamic Saturation Redesign}

\section{A. Main Result on Stubborn Redesign}

The first problem we deal with is the dynamic saturation, or "stubborn", [6], redesign of observer (4) given by

$$
\begin{aligned}
\dot{z} & =\varphi(z, u)+G \kappa\left(z, \operatorname{sat}_{\sigma}(y-h(\hat{x}))\right), \\
\dot{\sigma} & =-\Lambda \sigma+\Theta|y-h(\hat{x})|_{\mathrm{cw}}, \\
\hat{x} & =\psi(z)
\end{aligned}
$$

\footnotetext{
${ }^{1}$ The reader is referred to [30], [41] and references therein for more details about the notion of ISS and the existence of ISS-Lyapunov functions.

${ }^{2}$ With respect to [6], we use here separate saturation thresholds for the different output channels in order to improve responses since, when an outlier affects one channel, only the corresponding saturation level is influenced.
}

where $(z, \sigma) \in \mathbb{R}^{\varrho} \times \mathbb{R}_{\geq 0}^{m}$ is the state of the stubborn redesigned observer and $\Lambda:=\operatorname{diag}\left(\lambda_{1}, \ldots, \lambda_{m}\right)>0, \Theta:=\operatorname{diag}\left(\theta_{1}, \ldots, \theta_{m}\right)>$ 0 are design parameters. It can be noted that observer (10) is obtained by saturating the output error $y-h(\hat{x})$ in (4) with a variable saturation level $\sigma$. This level is dynamically driven by the same output error $y-h(\hat{x})$. The motivation for such a construction is that of filtering, at the steady state, sporadic perturbations that may affect the measured output $y$. Since in nominal conditions both the output error $y-h(\hat{x})$ and the saturation level $\sigma$ converge asymptotically to zero, when an outlier occurs, the saturation limits its effect on the observer state $z$, which is therefore less perturbed. A detailed analysis of this phenomenon in the linear context is given below in Section III-B.

The next theorem shows that observer (10) preserves the ISS of the original observer (4), according to the definitions given in Properties 1, 2.

Theorem 1 Consider system (3) satisfying Assumption 1. Suppose observer (4) is an ISS observer enjoying Properties 1 and 2. Let $\lambda_{i}>0, i=1, \ldots, m$ be fixed. Then, there exist $\theta_{i}^{*}>0, i=$ $1, \ldots, m$, such that, for any $\theta_{i}>\theta_{i}^{*}, i=1, \ldots, m$, observer (10) is an ISS observer for system (3) enjoying Properties 1 and 2.

Proof: In order to establish the result, we need to show the existence of an ISS-Lyapunov function for the perturbed-injection version of observer (10), which can be written in the compact form

$$
\begin{aligned}
\dot{z}_{e} & =\varphi_{e}\left(z_{e}, u\right)+G_{e} \kappa_{e}\left(z_{e}, y-h(\hat{x})\right)+G_{e} d_{e}, \\
\hat{x} & =\psi_{e}\left(z_{e}\right),
\end{aligned}
$$

with augmented state $z_{e}:=(z, \sigma) \in \mathbb{R}^{\varrho} \times \mathbb{R}_{\geq 0}^{m}$, disturbance $d_{e}:=\left(d, d_{\sigma}\right) \in \mathbb{R}^{\rho+m}$, and functions $\varphi_{e}\left(z_{e}, u\right):=(\varphi(z, u),-\Lambda \sigma)$, $\psi_{e}\left(z_{e}\right):=\psi(z), G_{e}:=\left[\begin{array}{cc}G & 0 \\ 0 & I\end{array}\right]$,

$$
\kappa_{e}\left(z_{e}, y-h(\hat{x})\right):=\left(\begin{array}{c}
\kappa\left(z, \operatorname{sat}_{\sigma}(y-h(\hat{x}))\right) \\
\Theta|y-h(\hat{x})|_{\mathrm{cw}}
\end{array}\right) .
$$

First, we see that functions $G_{e}, \kappa_{e}$ verify (6). In particular, to prove the second bound, recall that, by using the properties of $\kappa,(2)$ and the global Lipschitz property of $\operatorname{sat}_{\sigma}$, we have

$$
\begin{aligned}
& \left|\kappa_{e}\left(z_{e}, y_{1}\right)-\kappa_{e}\left(z_{e}, y_{2}\right)\right| \\
& \leq\left|\kappa\left(z, \operatorname{sat}_{\sigma}\left(y_{1}\right)\right)-\kappa\left(z, \operatorname{sat}_{\sigma}\left(y_{2}\right)\right)\right|+\left|\Theta\left(\left|y_{1}\right|_{\mathrm{cw}}-\left|y_{2}\right|_{\mathrm{cw}}\right)\right| \\
& \leq \bar{\kappa}_{e}\left|y_{1}-y_{2}\right|
\end{aligned}
$$

where $\bar{\kappa}_{e}:=\bar{\kappa}+\sqrt{m}|\Theta|$, with $\bar{\kappa}$ given in Property 1. In order to define an ISS-Lyapunov function $V_{e}$ for (11) according to Property 2, we first rewrite the $z_{e}$-dynamics in (11) as

$$
\begin{aligned}
\dot{z} & =\varphi(z, u)+G \kappa(z, \gamma)+G(\delta+d) \\
\dot{\sigma} & =-\Lambda \sigma+\Theta|\gamma|_{\mathrm{cw}}+\Theta \tilde{\gamma}+d_{\sigma}
\end{aligned}
$$

where we have defined the following compact notation

$$
\begin{aligned}
& \gamma:=h(x)-h(\psi(z)), \quad \bar{\gamma}:=\gamma+v, \quad \tilde{\gamma}:=|\bar{\gamma}|_{\mathrm{cw}}-|\gamma|_{\mathrm{cw}}, \\
& q:=\kappa\left(z, \operatorname{sat}_{\sigma}(\gamma)\right)-\kappa(z, \gamma), \\
& \tilde{q}:=\kappa\left(z, \operatorname{sat}_{\sigma}(\bar{\gamma})\right)-\kappa\left(z, \operatorname{sat}_{\sigma}(\gamma)\right), \quad \delta:=q+\tilde{q} .
\end{aligned}
$$

Using (1), the sector properties and global Lipschitz properties of the saturation and absolute value functions, the properties of $\kappa$, and the quantities in (14), it follows that

$$
\begin{gathered}
|q| \leq \bar{\kappa}\left|\operatorname{sat}_{\sigma}(\gamma)-\gamma\right| \leq \bar{\kappa}|\gamma|, \\
|\tilde{q}| \leq \bar{\kappa}|v|, \quad\left|\tilde{\gamma}_{i}\right| \leq\left|v_{i}\right| \leq|v| .
\end{gathered}
$$

According to the statement of the theorem, let $\lambda_{i}, i=1, \ldots, m$ be fixed and select $\theta_{i}^{*}, \eta>0$ as

$$
\theta_{i}^{*}:=\lambda_{i}+\frac{c_{d} \bar{\kappa}}{\eta}, \quad \eta:=\frac{c}{2 m\left(\ell_{1}+\ell_{d} \bar{\kappa} \ell_{0}\right)},
$$


with $c, c_{d}$ given in Property 1 and $\ell_{0}, \ell_{1}, \ell_{d}$ given in Property 2 . Then, fix any $\theta_{i}>\theta_{i}^{*}$ for $i=1, \ldots, m$, and let $\zeta_{i}>0, i=1, \ldots, m$, be chosen such that

$$
\sum_{i=1}^{m} \zeta_{i}\left(\theta_{i} \ell_{0}+\ell_{1}+\ell_{d} \bar{\kappa} \ell_{0}\right)<\frac{c}{2} .
$$

Note that condition (17) may always be satisfied by choosing $\zeta_{i}$ small enough. Bounds (16) and (17) will be then used below in the Lyapunov analysis. We define the ISS-Lyapunov function for (11) as

$$
\begin{aligned}
V_{e}(x, z, \sigma) & := \\
V(x, z) & +\sum_{i=1}^{m}\left(\zeta_{i} \sigma_{i}+\left(\zeta_{i}+\eta\right) \max \left\{\left|\gamma_{i}\right|-\sigma_{i}, 0\right\}\right),
\end{aligned}
$$

where $V$ comes from the ISS properties of (4) and satisfies Properties 1,2 , and the parameters $\zeta_{i}>0, i=1, \ldots, m$, and $\eta>0$ have been defined above. Note that, in view of the dynamics of $\sigma_{i}$ in (10), we have $\sigma(t) \in \mathbb{R}_{>0}^{m}$ for all $t \geq 0$ for any $\sigma(0) \in \mathbb{R}_{>0}^{m}$. As a consequence, we can use indifferently $\sigma_{i}=\left|\sigma_{i}\right|$. Due to the properties of $V, V_{e}$ in (18) can be lower bounded as follows

$$
V_{e}(x, z, \sigma) \geq V(x, z) \geq \underline{\alpha}(|x-\psi(z)|) .
$$

This shows that the lower bound in (7) is satisfied with $\underline{\alpha}_{e}(s)=\underline{\alpha}(s)$ for any $s \in \mathbb{R}_{>0}$. To prove the upper bound of (7), define $\psi_{e}^{-R}(x)=$ $\left(\psi^{-R}(x), 0\right)$, which satisfies

$$
\left|\psi_{e}^{-R}(x)-z_{e}\right|=\sqrt{\left|\psi^{-R}(x)-z\right|^{2}+|\sigma|^{2}} .
$$

Then, by using (1) and the output-growth condition on $V$ from Property 1, we can upper bound $V_{e}$ as follows

$$
\begin{aligned}
& V_{e}(x, z, \sigma) \\
& \leq V(x, z)+\sum_{i=1}^{m}(\bar{\zeta}+\eta)\left(\sigma_{i}+\left|\gamma_{i}\right|\right) \\
& \leq V(x, z)+(\bar{\zeta}+\eta) \sqrt{m}\left(|\sigma|+\ell_{0} V(x, z)\right) \\
& \left.\leq(\bar{\zeta}+\eta) \sqrt{m}|\sigma|+\left(1+(\bar{\zeta}+\eta) \sqrt{m} \ell_{0}\right) \bar{\alpha}\left(\left|\psi^{-R}(x)-z\right|\right)\right),
\end{aligned}
$$

where $\bar{\zeta}:=\max _{i}\left\{\zeta_{i}\right\}$. Now, for any $s_{1} \in \mathbb{R}^{n}, s_{2} \in \mathbb{R}^{m}, a, b>0$, and $\bar{\alpha} \in \mathcal{K}_{\infty}$, there always exists $\bar{\alpha}_{1} \in \mathcal{K}_{\infty}$ such that

$$
\begin{aligned}
a\left|s_{1}\right|+b \bar{\alpha}\left(\left|s_{2}\right|\right) & \leq \bar{\alpha}_{1}\left(\left|s_{1}\right|+\left|s_{2}\right|\right) \leq \bar{\alpha}_{1}\left(\sqrt{2} \sqrt{\left|s_{1}\right|^{2}+\left|s_{2}\right|^{2}}\right) \\
& \leq \bar{\alpha}_{1}\left(\sqrt{2}\left|\left(s_{1}, s_{2}\right)\right|\right) .
\end{aligned}
$$

Therefore, by combining the last inequality with (20), (21), we conclude that there exists $\bar{\alpha}_{e} \in \mathcal{K}_{\infty}$ such that

$$
V_{e}(x, z, \sigma) \leq \bar{\alpha}_{e}\left(\left|\psi_{e}^{-R}(x)-z_{e}\right|\right) .
$$

By combining (19) and (22), we verify that (7) is satisfied and therefore the function $V_{e}$ defined in (18) is a candidate ISS-Lyapunov function.

Before computing the Dini derivative of $V_{e}$ along the solutions to (13), we first verify that $V_{e}$ satisfies the output growth condition of Property 2 , since such bounds will be used to show (8). First note that the output function $h(\hat{x})$ of observer (13) is the same as that of (4) since the redesign involves only the output injection term. Therefore, according to (14) and by using inequality (9a) and (19), we obtain, for system (3) and observer (10),

$$
|\gamma|=|h(x)-h(\hat{x})| \leq \ell_{0} V(x, z) \leq \ell_{0} V_{e}(x, z, \sigma) .
$$

Then, using the definition of $\delta$ in (14) together with the bounds in (15) and (23), we have

$$
|\delta| \leq|q|+|\tilde{q}| \leq \bar{\kappa}|\gamma|+\bar{\kappa}|v| \leq \bar{\kappa} \ell_{0} V(x, z)+\bar{\kappa}|v| .
$$

We may then bound $\left|D^{+} \gamma\right|$, with $\gamma$ defined in (14), along the solutions to (13). In particular, using (9b), (19), and (24), we obtain

$$
\begin{aligned}
\left|D^{+} \gamma\right| \leq & \ell_{1} V(x, z)+\ell_{v}|v|+\ell_{w}|w|+\ell_{d}|\delta+d| \\
\leq & \left(\ell_{1}+\ell_{d} \ell_{0} \bar{\kappa}\right) V_{e}(x, z, \sigma) \\
& \quad+\left(\ell_{v}+\ell_{d} \bar{\kappa}\right)|v|+\ell_{w}|w|+\ell_{d}\left|d_{e}\right|,
\end{aligned}
$$

where the last step follows from $|d| \leq\left|d_{e}\right|$. In view of (23) and (25), we conclude that the Lyapunov function $V_{e}$ satisfies the output growth condition in Property 2.

Now, we need to show that inequality (8) holds for $V_{e}$, namely we need to analyze its Dini derivative along the solutions of (13). To this end, let us denote with $\mathcal{I} \subseteq\{1, \ldots, m\}$ the subset of indexes $i$ for which $\sigma_{i} \geq\left|\gamma_{i}\right|$, and with $\mathcal{J}=\{1, \ldots, m\} \backslash \mathcal{I}$ the set of indexes $j$ for which $\sigma_{j}<\left|\gamma_{j}\right|$. The derivative of $V_{e}$, computed from (8) and (13), and the first inequality in (25), satisfies

$$
\begin{aligned}
& D^{+} V_{e} \leq-c V(x, z)+c_{d}|\delta+d|+c_{v}|v|+c_{w}|w| \\
& \quad+\sum_{i \in \mathcal{I}} \zeta_{i}\left(-\lambda_{i} \sigma_{i}+\theta_{i}\left|\gamma_{i}\right|+\theta_{i}\left|\tilde{\gamma}_{i}\right|+\left|d_{\sigma_{i}}\right|\right) \\
& \quad+\sum_{j \in \mathcal{J}} \eta\left(\lambda_{j} \sigma_{j}-\theta_{j}\left|\gamma_{j}\right|+\theta_{j}\left|\tilde{\gamma}_{j}\right|+\left|d_{\sigma_{j}}\right|\right) \\
& \quad+\sum_{j \in \mathcal{J}}\left(\zeta_{j}+\eta\right)\left(\ell_{1} V(x, z)+\ell_{v}|v|+\ell_{w}|w|+\ell_{d}|\delta+d|\right),
\end{aligned}
$$

where we used the second bound in (9) and relation $D^{+}\left|\gamma_{i}\right| \leq$ $\left|D^{+} \gamma_{i}\right| \leq\left|D^{+} \gamma\right|$. By using the property of $\delta$ in (14), (15) and the properties of $\kappa, \mathcal{I}$ and $\mathcal{J}$, from (24) it follows that

$$
\begin{aligned}
|\delta| & \leq|q|+|\tilde{q}| \\
& \leq \bar{\kappa} \sum_{i \in \mathcal{I}}\left|\gamma_{i}\right|+\bar{\kappa} \sum_{j \in \mathcal{J}}\left|\gamma_{j}\right|+|\tilde{q}| \\
& \leq \bar{\kappa} \sum_{j \in \mathcal{J}}\left|\gamma_{j}\right|+\bar{\kappa}|v| .
\end{aligned}
$$

Using the previous bound and (15), together with (9) and (24), inequality (26) implies

$$
\begin{aligned}
D^{+} & V_{e} \overbrace{\left(c-\sum_{i \in \mathcal{I}} \zeta_{i} \theta_{i} \ell_{0}-\sum_{j \in \mathcal{J}}\left(\zeta_{j}+\eta\right)\left(\ell_{1}+\ell_{d} \bar{\kappa} \ell_{0}\right)\right.}^{\varepsilon_{V} V}) V(x, z) \\
& \overbrace{-\sum_{i \in \mathcal{I}} \zeta_{i} \lambda_{i} \sigma_{i}-\sum_{j \in \mathcal{J}}\left(\eta \theta_{j}\left|\gamma_{j}\right|-\eta \lambda_{j} \sigma_{j}-c_{d} \bar{\kappa}\left|\gamma_{j}\right|\right)}^{\pi_{\sigma}} \\
& +\left(c_{v}+c_{d} \bar{\kappa}+\sum_{i \in \mathcal{I}} \zeta_{i} \theta_{i}\right. \\
& +\left(c_{w}+\sum_{j \in \mathcal{J}}\left(\zeta_{j}+\eta\right) \ell_{w}\right)|w| \\
& +\left(c_{d}+\sum_{j \in \mathcal{J}}\left(\zeta_{j}+\eta\right) \ell_{d}\right)|d|+\sum_{i \in \mathcal{I}} \zeta_{i}\left|d_{\sigma_{i}}\right|+\sum_{j \in \mathcal{J}} \eta\left|\ell_{\sigma_{j}}\right| .
\end{aligned}
$$

Note that, in light of the selection of $\zeta_{i}, i=1, \ldots, p$ and $\eta$ according to (16) and (17), the following inequality holds

$$
\varepsilon_{V}=c-\sum_{i \in \mathcal{I}} \zeta_{i} \theta_{i} \ell_{0}-\sum_{j \in \mathcal{J}}\left(\zeta_{j}+\eta\right)\left(\ell_{1}+\ell_{d} \bar{\kappa} \ell_{0}\right)>0
$$

for any possible (finite) combination of sets $\mathcal{I}, \mathcal{J}$. Moreover, since $\theta_{i}>\theta_{i}^{*}$ and by the choice of $\theta_{i}^{*}$ in (16), we also get $\eta\left(\theta_{j}-\lambda_{j}\right)>$ $c_{d} \bar{\kappa}$. This provides, for some $\varepsilon_{\sigma}>0$,

$$
\begin{aligned}
\pi_{\sigma} & \leq-\sum_{i \in \mathcal{I}} \zeta_{i} \lambda_{i} \sigma_{i}+\sum_{j \in \mathcal{J}}-\eta \theta_{j}\left|\gamma_{j}\right|+\eta \lambda_{j} \sigma_{j}+c_{d} \bar{\kappa}\left|\gamma_{j}\right| \\
& \leq-\varepsilon_{\sigma} \sum_{i \in \mathcal{I}} \sigma_{i}-\varepsilon_{\sigma} \sum_{j \in \mathcal{J}} \sigma_{j}=-\varepsilon_{\sigma} \sum_{k=1}^{m} \sigma_{k}=-\varepsilon_{\sigma}|\sigma|_{1},
\end{aligned}
$$


where we have used both $\sigma_{j}<\left|\gamma_{j}\right|$ and $-\left|\gamma_{j}\right|<-\sigma_{j}$ for all $j \in \mathcal{J}$, and the fact that $\sigma_{i} \geq 0$ for all $i=1, \ldots, m$. As a consequence, combining the above bounds with (27), there exists $\bar{\delta}>0$ such that

$$
D^{+} V_{e} \leq-\varepsilon_{V} V(x, z)-\varepsilon_{\sigma}|\sigma|_{1}+\bar{\delta}|v|+\bar{\delta}|w|+\bar{\delta}\left|d_{e}\right|,
$$

for all $(x, z, \sigma) \in \mathcal{X} \times \mathcal{Z} \times \mathbb{R}_{>0}^{m}$. Moreover, using the last inequality with (1) and (19), we obtain, for some $\varepsilon_{0}>0$,

$$
D^{+} V_{e} \leq-\varepsilon_{0} V_{e}(x, z, \sigma)+\bar{\delta}(|v|+|w|+|d|)
$$

for all $(x, z, \sigma) \in \mathcal{X} \times \mathcal{Z} \times \mathbb{R}_{\geq 0}^{m}$. With this, it follows that observer (10) is ISS and function $V_{e}$ is an ISS-Lypaunov function satisfying Property 1.

\section{B. Performance Analysis of the Saturation Redesign}

The effect of the dynamic saturation in (10) is that of mitigating the effect of impulsive disturbances (outliers) of $v$ on the estimation error $\hat{x}-x$, thus improving the transient behavior, at the cost of slowing down the convergence rate, yet still preserving the asymptotic estimation. Although a complete noise analysis is hard to carry out in the nonlinear framework (see, among others, the discussions in [12]), some results can be proven if (3), (4) are linear and $y \in \mathbb{R}$ as in [6], where no performance characterization was given. In particular, consider the linear system

$$
\dot{x}=A x+B u, \quad y=C x+v
$$

where $x \in \mathbb{R}^{n}$ is the state, $y \in \mathbb{R}$ is the measured output, $v$ is the measurement noise, and $(A, C)$ is an observable pair. The observer for (29) is selected as

$$
\dot{\hat{x}}=A \hat{x}+B u+L(y-C \hat{x})
$$

where $\hat{x} \in \mathbb{R}^{n}$ is the estimate and $L$ is such that $A-L C$ is Hurwitz. We denote the estimation error provided by observer (30) as $\tilde{x}_{0}:=$ $\hat{x}-x$, thus obtaining

$$
\dot{\tilde{x}}_{0}=(A-L C) \tilde{x}_{0}+L v .
$$

By following the design proposed in Theorem 1, we consider also the stubborn redesigned observer

$$
\begin{aligned}
\dot{\hat{x}} & =A \hat{x}+B u+L \operatorname{sat}_{\sigma}(y-C \hat{x}), \\
\dot{\sigma} & =-\lambda \sigma+\theta|y-C \hat{x}|,
\end{aligned}
$$

with $\sigma \in \mathbb{R}_{\geq 0}, \lambda, \theta>0$. Similarly, we denote the estimation error of observer (32) as $\tilde{x}_{\text {sat }}:=\hat{x}-x$. In these coordinates, we obtain the following error dynamics

$$
\begin{aligned}
\dot{\tilde{x}}_{\mathrm{sat}} & =A \tilde{x}_{\mathrm{sat}}+L \operatorname{sat}_{\sigma}\left(v-C \tilde{x}_{\mathrm{sat}}\right) \\
\dot{\sigma} & =-\lambda \sigma+\theta\left|v-C \tilde{x}_{\mathrm{sat}}\right| .
\end{aligned}
$$

To comparatively characterize the effect of impulsive disturbances $v$ on the two observers (30) and (32), we model $v$ as a piecewise constant perturbation of the form

$$
v=\delta_{\tau}(t)= \begin{cases}\frac{1}{\tau} & 0 \leq t \leq \tau \\ 0 & t>\tau\end{cases}
$$

where $\delta_{\tau}(t)$ converges to the Dirac delta function as $\tau \rightarrow 0^{+}$. The following result establishes extreme improvements of the error response $\tilde{x}_{\text {sat }}$ over $\tilde{x}_{0}$ as $\tau \rightarrow 0^{+}$by focusing on $\left|\tilde{x}_{\text {sat }}(\tau)\right|$ and $\left|\tilde{x}_{0}(\tau)\right|$. This is relevant because for $t>\tau$ we have $\delta_{\tau}(t)=0$ and both observers evolve with $v=0$.

Proposition 1 Consider (29) with $A \in \mathbb{R}^{n \times n}$ nonsingular and denote by $\tilde{x}_{0}$ and $\tilde{x}_{\mathrm{sat}}$ the solutions, respectively, to (31) and (33) with $\tilde{x}_{0}(0)=\tilde{x}_{\text {sat }}(0)=0, \sigma(0)=0$, and $v$ given by (34). As $\tau$ tends to $0^{+}$, the following holds

$$
\frac{\left|\tilde{x}_{\text {sat }}(\tau)\right|}{\left|\tilde{x}_{0}(\tau)\right|} \leq \tau \theta \frac{1+o(\tau)}{1-o(\tau)}
$$

where o $(\tau)$ denotes small terms in $\tau^{r}, r \geq 1$. Furthermore, for each $\tau \geq 0,\left|\tilde{x}_{\text {sat }}(t)\right|$ converges to zero as $\tau \rightarrow 0^{+}$.

Proof: First compute

$$
\begin{aligned}
\tilde{x}_{0}(\tau) & =\int_{0}^{\tau} e^{(A-L C)(\tau-s)} L \delta_{\tau}(s) d s \\
& =\left(e^{(A-L C) \tau}-I\right)(A-L C)^{-1} L \frac{1}{\tau} .
\end{aligned}
$$

As a first approximation, for $\tau \rightarrow 0^{+}$, we use the Taylor expansion to compute $e^{(A-L C) \tau}=I+(A-L C) \tau+o\left(\tau^{2}\right)$, where, with some abuse of notation, we denote with $o(\tau)$ higher-order infinitesimal terms in $\tau$ and these terms may be a scalar or a matrix. Therefore, we obtain

$$
\left|\tilde{x}_{0}(\tau)\right|=\frac{1}{\tau}|L|\left(\tau+o\left(\tau^{2}\right)\right) \geq|L|(1-o(\tau)) .
$$

Similarly, we compute $\tilde{x}_{\text {sat }}(\tau)$. We have

$$
\tilde{x}_{\mathrm{sat}}(\tau)=\int_{0}^{\tau} e^{A(\tau-s)} L \sigma(s) d s,
$$

with $\sigma(s), s \in[0, \tau]$, given by

$$
\sigma(s)=\int_{0}^{s} e^{-\lambda(s-r)} \theta\left|\delta_{\tau}(r)-C \tilde{x}_{\mathrm{sat}}(r)\right| d r .
$$

By integrating (38) from $\sigma(0)=0$ and using (34), we obtain

$$
\begin{aligned}
& \sigma(s) \leq \frac{\theta}{\lambda}\left(1-e^{-\lambda s}\right)\left(\frac{1}{\tau}+c\right) \leq \theta\left(s+o\left(s^{2}\right)\right)\left(\frac{1}{\tau}+c\right) \\
& \leq \theta(1+o(\tau))(1+\tau c) \leq \theta(1+\tau c+o(\tau)),
\end{aligned}
$$

which holds for all $s \in[0, \tau]$, where we have approximated $e^{-\lambda \tau}$ by $1-\lambda \tau+o\left(\tau^{2}\right)$ for small $\tau$ and where $c=\sup _{s \in[0, \tau]}\left|C \tilde{x}_{\text {sat }}(s)\right|$. By using (37), we further get

$$
\begin{aligned}
\left|\tilde{x}_{\text {sat }}(\tau)\right| & \leq\left|\int_{0}^{\tau} e^{A(\tau-s)} L\right| \sigma(s)|d s| \\
& \leq\left|\left(e^{A \tau}-I\right) A^{-1} L \theta(1+\tau c+o(\tau))\right| \\
& \leq\left|\left(A \tau+o\left(\tau^{2}\right)\right) A^{-1} L \theta(1+\tau c+o(\tau))\right| \\
& \leq \tau \theta|L|(1+o(\tau)) .
\end{aligned}
$$

for $\tau \rightarrow 0^{+}$. In light of (36) and (39), we conclude that inequality (35) holds. Finally, when $\tau \rightarrow 0^{+}$, namely when $\delta_{\tau}$ tends to a Dirac impulse, by using (39), it follows that $\left|\tilde{x}_{\text {sat }}(\tau)\right| \rightarrow 0$ and in particular $\tilde{x}_{\text {sat }}(t) \rightarrow 0$ for all $t \geq 0$.

While Proposition 1 illustrates the advantages of (33) versus (31) in terms of dynamic response, we prove below that the static response, as measured by the disturbance-to-error DC gain, is not worse in (33) when $\theta \geq \lambda$ (which always holds under (16)).

Proposition 2 Assume that $A-L C$ is Hurwitz. For any $\theta \geq \lambda$, the disturbance-to-error DC gains of observers (30) and (32) associated to a constant $v$ coincide.

Proof: The equilibrium $\tilde{x}_{0}^{\circ}$ of (31) for any constant $v(t)=\bar{v}$ for all $t \geq 0$ is given by $\tilde{x}_{0}^{\circ}=-(A-L C)^{-1} L \bar{v}$. Concerning dynamics (33), the equilibrium $\sigma^{\circ}$ of the $\sigma$-dynamics is given by $\sigma^{\circ}=\frac{\theta}{\lambda} \mid \bar{v}-$ $C \tilde{x}_{\text {sat }}^{\circ}$, where $\tilde{x}_{\text {sat }}^{\circ}$ denotes the equilibrium of the $\tilde{x}_{\text {sat }}$-dynamics. For $\theta \geq \lambda$, it follows that $\sigma \geq\left|\bar{v}-C \tilde{x}_{\text {sat }}^{\circ}\right|$, therefore $\operatorname{sat}_{\sigma}\left(\bar{v}-C \tilde{x}_{\text {sat }}^{\circ}\right)=$ $\bar{v}-C \tilde{x}_{\text {sat }}^{\circ}$. As a consequence, in the $\tilde{x}_{\text {sat }}$ dynamics we obtain again $\tilde{x}_{\text {sat }}^{\circ}=-(A-L C)^{-1} L \bar{v}$, namely $\tilde{x}_{\text {sat }}^{\circ}=\tilde{x}_{0}^{\circ}$. 


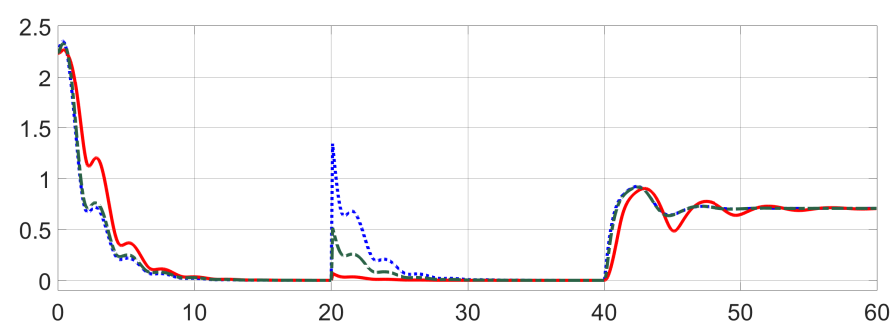

Fig. 1: Effect of the dynamic saturation redesign in the presence of outliers: $\left|\tilde{x}_{0}(t)\right|$ (in dotted blue) of error dynamics (31), $\left|\tilde{x}_{\text {sat }}(t)\right|$ of error dynamics (33) with $\lambda=\theta=10$ (in dashed green) and with $\lambda=\theta=1$ (in red).

As a simple illustration of Propositions 1 and 2, we have analyzed the case in which system (29) is selected as $A=\left[\begin{array}{cc}0 & 1 \\ -1 & 0\end{array}\right], B=\left[\begin{array}{l}0 \\ 0\end{array}\right]$, $C=\left[\begin{array}{ll}1 & 0\end{array}\right]$ and observer (30) has gain $L=\left[\begin{array}{l}1 \\ 1\end{array}\right]$. In order to show the effect of the saturation redesign, we consider two different choices of the parameters: $\lambda=\theta=1$ and $\lambda=\theta=10$. The initial conditions are selected as $x(0)=(0,1)$ and $\hat{x}(0)=(1,-1)$ for all the observers. An impulse of the form (34) with $\tau=0.1$ is applied at time $t=20$. A constant disturbance of unitary amplitude is then applied at time $t=40$. Fig. 1 shows the evolution of the norm $\left|\tilde{x}_{0}(t)\right|$ of the error dynamics (31) and the norms $\left|\tilde{x}_{\text {sat }}(t)\right|$ of the error dynamics (33) for the two different choices of the parameters. When $t \in[0,20]$ we can see that the presence of the saturation slightly deteriorates the transient response when $\lambda$ is selected small (red curve). Then, between $t \in[20,40]$ we can observe the effect of the outlier. The maximum value is reached at $t=20+\tau$, where we obtained $\left|\tilde{x}_{0}(\tau)\right|=1.343,\left|\tilde{x}_{\text {sat }}(\tau)\right|=0.516$ for $\theta=10$ and $\left|\tilde{x}_{\text {sat }}(\tau)\right|=0.068$ for $\theta=1$. These values confirm bound (35) since $\left|\tilde{x}_{\text {sat }}(\tau)\right| /\left|\tilde{x}_{0}(\tau)\right|=0.384 \leq \theta \tau=1$ for $\theta=10$ and $\left|\tilde{x}_{\text {sat }}(\tau)\right| /\left|\tilde{x}_{0}(\tau)\right|=0.051 \leq \theta \tau=0.1$ for $\theta=1$. Finally, in $t \in[40,60]$ a constant disturbance of unitary amplitude is applied, namely $v(t)=1$ for all $t \geq 40$. The three observers asymptotically converge to the same value, confirming that their DC gain is the same, as stated in Proposition 2.

We finally remark that, although the bounds and the simulations of this section refer to the case of linear systems and observers, similar results can be numerically appreciated with nonlinear systems/estimators, as shown in [9].

\section{User Guidelines for Saturation Parameter Tuning}

Theorem 1 provides a sufficient result for a suitable choice of parameters $\Lambda, \Theta$ in (10). Such a result is in general conservative as it imposes a large lower-bound for the value of $\Theta$, see (16). The simulation in Fig. 1 shows the influence of the two parameters highlighting that a small $\Lambda$ potentially deteriorates the transient responses, while a small $\Theta$ improves the rejection of impulsive disturbances. As a consequence, from a practical point of view, the tuning of $\Lambda, \Theta$ based on the simulation outcomes is much more effective for the achieved performances.

We conclude that, to optimize the tuning of the parameters, one should select values of $\Lambda$ of the same order of magnitude as the decay rate of the estimation error of the original observer, in order not to deteriorate too much the transient response, and, at the same time, choose $\Theta$ to minimize the ratio $\Lambda / \Theta$ so as to improve the sensitivity to measurement noise.

\section{Dynamic DeAd-Zone REDEsIGN}

\section{A. Main Result on Dead-Zone Redesign}

In line with [24], we discuss here the use of a dynamic dead-zone redesign of observer (4) given by

$$
\begin{aligned}
\dot{z} & =\varphi(z, u)+G \kappa\left(z, \mathrm{dz}_{\sigma}(y-h(\hat{x}))\right), \\
\dot{\sigma} & =-\Lambda \sigma+\Theta|y-h(\hat{x})|_{\mathrm{cw}}, \\
\hat{x} & =\psi(z)
\end{aligned}
$$

where $(z, \sigma) \in \mathbb{R}^{\varrho} \times \mathbb{R}_{>0}^{m}$ is the state of the dead-zone redesigned observer and $\Lambda:=\operatorname{diag}\left(\bar{\lambda}_{1}, \ldots, \lambda_{m}\right)>0, \Theta:=\operatorname{diag}\left(\theta_{1}, \ldots, \theta_{m}\right) \geq$ 0 are design parameters. Paralleling the stubborn redesign in (10), observer (40) is obtained by "dead-zonating" the output error $y-h(\hat{x})$ in (4) with a variable dead-zone level $\sigma$. The level $\sigma$ is driven by the same output error $|y-h(\hat{x})|_{\mathrm{cw}}$. From the peculiar shape of the dead-zone function, the motivation for this redesign is that of "trimming" the effect of persistent bounded sensor noises. A more precise analysis of the effect of the dead-zone in the linear context is given below in Section IV-B.

Note that, despite the presence of the dead-zone, the use of a variable threshold allows retaining the asymptotic convergence to zero of the estimation error in nominal conditions. This is formally stated in the next theorem, where we show that, similar to the stubborn redesign of the previous section, also the dead-zone redesigned observer (40) preserves the ISS of the original observer (4), according to the definitions given in Properties 1, 2 .

Theorem 2 Consider system (3) satisfying Assumption 1. Suppose observer (4) is an ISS observer enjoying Properties 1 and 2. Let $\theta_{i} \geq 0, i=1, \ldots, m$ be fixed. Then, there exist $\lambda_{i}^{*}>0, i=$ $1, \ldots, m$, such that, for any $\lambda_{i}>\lambda_{i}^{*}, i=1, \ldots, m$, observer (40) is an ISS observer for system (3) enjoying Properties 1 and 2.

Proof: First, let us rewrite the perturbed-injection version of observer (40) as in (11) and use the subscript $e$ for $\varphi_{e}, G_{e}, \psi_{e}, \kappa_{e}, d_{e}, V_{e}$ as in Section III. Function $\kappa_{e}$ is now given by

$$
\kappa_{e}\left(z_{e}, y-h(\hat{x})\right):=\left(\begin{array}{c}
\kappa\left(z, \mathrm{dz}_{\sigma}(y-h(\hat{x}))\right) \\
\Theta|y-h(\hat{x})|_{\mathrm{cw}}
\end{array}\right)
$$

whereas $z_{e}, d_{e}, \varphi_{e}, G_{e}$, and $\psi_{e}$ are defined after (11). First, we see that functions $G_{e}, \kappa_{e}$ verify (6). In particular, to prove the right bound, recall that, by using (2) and the properties of $\kappa$ and of the dead-zone function, we have

$$
\begin{aligned}
& \left|\kappa_{e}\left(z_{e}, y_{1}\right)-\kappa_{e}\left(z_{e}, y_{2}\right)\right| \\
& \quad \leq\left|\kappa\left(z, \mathrm{dz}_{\sigma}\left(y_{1}\right)\right)-\kappa\left(z, \mathrm{dz}_{\sigma}\left(y_{2}\right)\right)\right|+\left|\Theta\left(\left|y_{1}\right|_{\mathrm{cw}}-\left|y_{2}\right|_{\mathrm{cw}}\right)\right| \\
& \quad \leq \bar{\kappa}_{e}\left|y_{1}-y_{2}\right|
\end{aligned}
$$

where $\bar{\kappa}_{e}=\bar{\kappa}+\sqrt{m}|\Theta|$, with $\bar{\kappa}$ given in Property 1 . In order to define an ISS-Lyapunov function $V_{e}$ for (11) satisfying Property 2, note that dynamics (40) can be rewritten by expressing (11) as in (13) with the new definitions

$$
\begin{aligned}
& \gamma:=h(x)-h(\psi(z)), \quad \tilde{\gamma}:=|\bar{\gamma}|_{\mathrm{cw}}-|\gamma|_{\mathrm{cw}}, \quad \bar{\gamma}:=\gamma+v, \\
& q:=\kappa\left(z, \mathrm{dz}_{\sigma}(\gamma)\right)-\kappa(z, \gamma), \\
& \tilde{q}:=\kappa\left(z, \mathrm{dz}_{\sigma}(\bar{\gamma})\right)-\kappa\left(z, \mathrm{dz}_{\sigma}(\gamma)\right), \quad \delta:=q+\tilde{q} .
\end{aligned}
$$

The quantities in (42) satisfy the following inequalities from the sector and global Lipschitz properties of the saturation, the absolute value, and $\kappa$ :

$$
\begin{gathered}
|q| \leq \bar{\kappa}\left|\mathrm{dz}_{\sigma}(\gamma)-\gamma\right| \leq \bar{\kappa}|\sigma|, \\
|\tilde{q}| \leq \bar{\kappa}|v|, \quad\left|\tilde{\gamma}_{i}\right| \leq\left|v_{i}\right| \leq|v|
\end{gathered}
$$


According to the statement of the theorem, let $\theta_{i}, i=1, \ldots, m$ be fixed, and first select some $\zeta_{i}>0$ satisfying

$$
\ell_{0} \sum_{i=1}^{m} \zeta_{i} \theta_{i}<c
$$

with $c$ given in Property 1 and $\ell_{0}$ given in Property 2 . Then, select $\lambda_{i}^{*}>0$ such that

$$
\lambda_{i}^{*} \zeta_{i}>c_{d} \bar{\kappa}, \quad \forall i=1, \ldots, m .
$$

We define the ISS-Lyapunov function for (40) as

$$
V_{e}(x, z, \sigma):=V(x, z)+\sum_{i=1}^{m} \zeta_{i} \sigma_{i} \leq V(x, z)+\bar{\zeta}|\sigma|_{1}
$$

where $\zeta_{i}>0, i=1, \ldots, m$, have been defined above and $\bar{\zeta}:=$ $\max \left\{\zeta_{i}\right\}$. Note that, in light of the property of the dynamics of $\sigma$ in (40), we have $\sigma(t) \in \mathbb{R}_{\geq 0}^{m}$ for all $t \geq 0$ for any $\sigma(0) \in \mathbb{R}_{\geq 0}^{m}$. Thus, we can write indifferently $\sigma_{i}=\left|\sigma_{i}\right|$. As in the proof of Theorem 1 , we define $\psi_{e}^{-R}(x):=\left(\psi^{-R}(x), 0\right)$. Therefore, we obtain

$$
\underline{\alpha}_{e}(|x-\psi(z)|) \leq V_{e}(x, z, \sigma) \leq \bar{\alpha}_{e}\left(\left|\psi_{e}^{-R}(x)-z_{e}\right|\right)
$$

where $\underline{\alpha}_{e}(s)=\underline{\alpha}(s)$ for any $s \in \mathbb{R}_{\geq 0}$, with $\underline{\alpha}$ given by Property 1 , and $\bar{\alpha}_{e} \in \mathcal{K}_{\infty}$ satisfying

$$
\bar{\alpha}\left(s_{1}\right)+\sqrt{m} \max \left\{\zeta_{i}\right\} s_{2} \leq \bar{\alpha}_{e}\left(\sqrt{2}\left|\left(s_{1}, s_{2}\right)\right|\right) .
$$

This shows that (7) holds and therefore function $V_{e}$ defined in (46) is a candidate ISS-Lyapunov function. Furthermore, it satisfies the output growth condition of Property 2. To see this, note that (23) applies also to this case. Concerning its Dini derivative, we may use the bounds in (43) to prove (24) and (25), thus showing the conditions of Property 2. We now focus on proving (8). In order to compute the Dini derivative of $V_{e}$ along the solutions to (13), (40), we use (9a) and (43) as follows

$$
\begin{aligned}
|\delta| & \leq|q|+|\tilde{q}| \leq \bar{\kappa}\left|\operatorname{sat}_{\sigma}(\gamma)\right|+\bar{\kappa}|v| \leq \bar{\kappa}|\sigma|+\bar{\kappa}|v|, \\
\left|\gamma_{i}\right| & \leq|\gamma| \leq \ell_{0} V(x, z) .
\end{aligned}
$$

Then, we obtain

$$
\begin{aligned}
D^{+} & V_{e}=D^{+} V+\sum_{i=1}^{m} \zeta_{i} D^{+} \sigma_{i} \\
\leq & -c V(x, z)+c_{d}|\delta+d|+c_{v}|v|+c_{w}|w| \\
& +\sum_{i=1}^{m} \zeta_{i}\left(-\lambda_{i} \sigma_{i}+\theta_{i}\left|\gamma_{i}\right|+\theta_{i}\left|\tilde{\gamma}_{i}\right|+\left|d_{\sigma_{i}}\right|\right) \\
\leq & -c V(x, z)+c_{d} \bar{\kappa}|\sigma|_{1} \\
& +\sum_{i=1}^{m} \zeta_{i}\left(-\lambda_{i} \sigma_{i}+\theta_{i} \ell_{0} V(x, z)+\theta_{i}|v|+\left|d_{\sigma_{i}}\right|\right) \\
& +c_{d}|d|+\left(c_{v}+c_{d} \bar{\kappa}\right)|v|+c_{w}|w| \\
\leq & -\varepsilon\left(V(x, z)+|\sigma|_{1}\right)+\bar{\delta}|v|+\bar{\delta}|w|+\bar{\delta}\left|d_{e}\right|
\end{aligned}
$$

for some $\varepsilon, \bar{\delta}>0$, and for all $(x, z, \sigma) \in \mathcal{X} \times \mathcal{Z} \times \mathbb{R}_{>0}^{m}$, where (43), (44), and (45) are used to derive the last inequality. From (48) and the right bound in (46), it follows that observer (40) is an ISS observer and function $V_{e}$ is an ISS-Lyapunov function satisfying Properties 1 and 2.

\section{B. Performance Analysis of the Dead-Zone Redesign}

As in Section III-B, we analyze here the effect of a constant perturbation $v(t)=\bar{v}$ for all $t \geq 0$ on the redesigned observer (40) for the single-output linear case. In particular, we consider once again the single-output linear system (29) where $(A, C)$ is an observable pair, interconnected to the linear observer (30), where $L$ is such that $A-L C$ is Hurwitz. Denoting the esimation error as $\tilde{x}_{0}:=\hat{x}-x$, we obtain the linear error dynamics (31), repeated here for convenience:

$$
\dot{\tilde{x}}_{0}=(A-L C) \tilde{x}_{0}+L v .
$$

By following the design proposed in Theorem 2, we consider then the dead-zone redesigned observer

$$
\begin{aligned}
\dot{\hat{x}} & =A \hat{x}+B u+L \mathrm{dz}_{\sigma}(y-C \hat{x}) \\
\dot{\sigma} & =-\lambda \sigma+\theta|y-C \hat{x}|
\end{aligned}
$$

with $\sigma \in \mathbb{R}_{\geq 0}, \lambda>0, \theta \geq 0$. Similarly, we denote the estimation error of observer (50) as $\tilde{x}_{\mathrm{dz}}:=\hat{x}-x$. In these coordinates, we obtain the error dynamics

$$
\begin{aligned}
\dot{\tilde{x}}_{\mathrm{dz}} & =A \tilde{x}_{\mathrm{dz}}+L \mathrm{dz}_{\sigma}\left(v-C \tilde{x}_{\mathrm{dz}}\right) \\
\dot{\sigma} & =-\lambda \sigma+\theta\left|v-C \tilde{x}_{\mathrm{dz}}\right| .
\end{aligned}
$$

By following the analysis in Section III-B, we compare the static responses, as measured by the disturbance-to-error DC gain, of the error dynamics (49) and (51). For this, we focus on the class of triplets $A, C, L$ such that $A$ is nonsingular and the gain $C A^{-1} L<1$. This condition is not overly restrictive and is enjoyed by all triplets $A, C, L$ such that both $A$ and $A-L C$ are Hurwitz, as illustrated by the next lemma.

Lemma 1 All the triplets $(A, C, L)$ such that $\operatorname{det}(A-$ $L C) \operatorname{det}(A)>0$, satisfy $C A^{-1} L<1$, in particular this condition holds if $A$ and $A-L C$ are both Hurwitz.

Proof: The condition $\operatorname{det}(A-L C) \operatorname{det}(A)>0$ implies $\operatorname{det}(A) \neq 0$, hence $A$ is invertible. By applying the matrix determinant lemma ${ }^{3}$ we compute $\operatorname{det}(A-L C)=\operatorname{det}\left(A\left(I-A^{-1} L C\right)\right)=$ $\operatorname{det}(A)\left(1-C A^{-1} L\right)$. As a consequence, if $\operatorname{det}(A-L C) \operatorname{det}(A)>$ 0 , then $1-C A^{-1} L>0$, which concludes the proof.

We may now state our main DC-gain result by establishing a desirable strict decrease of the DC gain of (50) as $\theta$ is increased from 0 (in this case the dead-zone is inactive and the DC gains are the same) to its maximum value.

Proposition 3 Suppose that $A-L C$ is Hurwitz. If $A$ is invertible and $C A^{-1} L<1$, then there exists a class $\mathcal{K}$ function $\tilde{k}$ such that, for any $\lambda>\theta \geq 0$, the disturbance-to-error DC gains $k_{0}, k_{\mathrm{dz}}$ of dynamics (49), respectively (51), satisfy

$$
\frac{k_{\mathrm{dz}}}{k_{0}}=1-\tilde{k}\left(\frac{\theta}{\lambda}\right) .
$$

Proof: The equilibrium $\tilde{x}_{0}^{\circ}$ of error dynamics (49) for a constant $v(t)=\bar{v}$ for all $t \geq 0$ is given by

$$
\tilde{x}_{0}^{\circ}=-(A-L C)^{-1} L \bar{v} .
$$

To compute the equilibrium $\left(\tilde{x}_{\mathrm{dz}}^{\circ}, \sigma^{\circ}\right)$ of error dynamics (51) for the same $v=\bar{v}$ constant, we first get $\sigma^{\circ}=\frac{\theta}{\lambda}\left|\bar{v}-C \tilde{x}_{\mathrm{dz}}^{\circ}\right|$. For any $\theta \leq \lambda$, we have $\sigma^{\circ} \leq\left|\bar{v}-C \tilde{x}_{\mathrm{dz}}^{\circ}\right|$, and hence, by the properties of the dead-zone function and the error dynamics (51) at the equilibrium, it follows that $A \tilde{x}_{\mathrm{dz}}+L\left(\bar{v}-C \tilde{x}_{\mathrm{dz}}\right) \varepsilon=0$ with $\varepsilon:=1-\theta / \lambda$. Rearranging, we get $\tilde{x}_{\mathrm{dz}}^{\circ}=-\varepsilon(A-L C \varepsilon)^{-1} L \bar{v}$ with $\varepsilon \in[0,1]$. Now, by using the Woodbury matrix identity ${ }^{4}$ we obtain

$$
\begin{aligned}
& \tilde{x}_{\mathrm{dz}}^{\circ}=-\varepsilon(A-L C \varepsilon)^{-1} L \bar{v} \\
& =-\left(A^{-1}+\frac{\varepsilon}{1-\varepsilon C A^{-1} L} A^{-1} L C A^{-1}\right) L \varepsilon \bar{v} \\
& =\frac{-\varepsilon \bar{v}}{1-\varepsilon C A_{\varepsilon}^{-1} L}\left(A^{-1} L-\varepsilon A^{-1} L C A^{-1} L+\varepsilon A^{-1} L C A^{-1} L\right) \\
& =-\frac{{ }^{-1}}{1-\varepsilon C A^{-1} L} A^{-1} L \bar{v}=-\alpha(\varepsilon) A^{-1} L \bar{v},
\end{aligned}
$$

${ }^{3}$ For $A$ invertible and $u, v$ column vectors, the matrix determinant lemma states that $\operatorname{det}\left(A+u v^{\top}\right)=\operatorname{det}(A) \operatorname{det}\left(1+v^{\top} A^{-1} u\right)$.

${ }^{4}$ Also known as matrix inversion lemma, the Woodbury matrix identity states that for $A$ invertible and $u, v$ column vectors, the following holds: $\left(A+u v^{\top}\right)^{-1}=A^{-1}-\frac{1}{1+v^{\top} A^{-1} u} A^{-1} u v^{\top} A^{-1}$. 


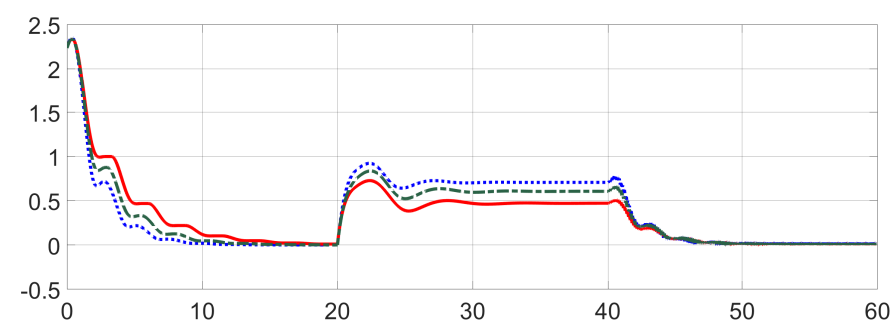

Fig. 2: Effect of the dynamic dead-zone redesign in the presence of outliers: $\left|\tilde{x}_{0}(t)\right|$ (in dotted blue) of error dynamics (49), $\left|\tilde{x}_{\mathrm{dz}}(t)\right|$ of error dynamics (51) with $\lambda=4, \theta=1$ (in dashed green) and with $\lambda=2, \theta=1$ (in red).

where $\alpha(\varepsilon):=\varepsilon /(1-a \varepsilon)$, with $a:=C A^{-1} L<1$ from the statement of the proposition. From the previous equation and (53) it follows that $\left|\tilde{x}_{0}^{\circ}\right|=\alpha(1)\left|A^{-1} L \bar{v}\right|$ and $\left|\tilde{x}_{\mathrm{dz}}^{\circ}\right|=\alpha(\varepsilon)\left|A^{-1} L \bar{v}\right|$, which provides

$$
\frac{k_{\mathrm{dz}}}{k_{0}}=\frac{\left|\tilde{x}_{\mathrm{dz}}^{\circ}\right|}{\left|\tilde{x}_{0}^{\circ}\right|}=\frac{\alpha(\varepsilon)}{\alpha(1)}=1-\tilde{k}(\tilde{\theta}),
$$

where we denote $\tilde{\theta}:=\theta / \lambda$. It is then immediate to determine the function $\tilde{k}$ in $(52)$ as $\tilde{k}(\tilde{\theta})=1-\alpha(1-\tilde{\theta}) / \alpha(1)=\tilde{\theta} /(1+a(\tilde{\theta}-1))$. To show that $\tilde{k} \in \mathcal{K}$ whenever $a<1$ and $\theta \in[0,1]$, note that $\tilde{k}(0)=0$ and the sign of its derivative, i.e., $\tilde{k}(\tilde{\theta})^{\prime}=(1-a) /(1+a(\tilde{\theta}-1))^{2}>0$.

We finally remark that, although the bounds derived in this section refer to the case of linear systems and observers, similar results can be observed in numerical simulations with nonlinear systems/observers, as shown in [23], [24].

As in Section III-B, simple simulation results are provided to illustrate Proposition 3. We select the matrices in (29), as $A=$ $\left[\begin{array}{cc}0 & 1 \\ -1 & 0\end{array}\right], B=\left[\begin{array}{l}0 \\ 0\end{array}\right], C=\left[\begin{array}{ll}1 & 0\end{array}\right]$, and the output injection gain $L=\left[\begin{array}{l}1 \\ 1\end{array}\right]$ for observer (30). In order to show the effect of the saturation redesign we consider two different parameters choices, namely $\lambda=4, \theta=1$ and $\lambda=2, \theta=1$. The initial conditions are selected as $x(0)=(0,1)$ and $\hat{x}(0)=(1,-1)$ for all the observers. Fig. 2, for $t \in[0,20]$, shows that the convergence of the estimation error in the absence of noise is guaranteed for all of the considered observers. The simulations illustrate that the dead-zone may deteriorate the transient response of the estimation error when the ratio $\theta / \lambda$ is too large. Then, a constant unitary measurement noise is applied at time $t=20$. The asymptotic value of the observer dynamics (49) is 0.707 , while those of the dead-zonated observers are 0.606 and 0.471 for $\lambda=4$ and $\lambda=2$, respectively. This result confirms the bounds of Proposition 3, as the DC gain is reduced by augmenting the ratio $\theta / \lambda$. Finally, from time $t=40$, we apply a high-frequency measurement noise of unitary magnitude modeled as $v(t)=\sin (50 t)$. From the simulations we compute the bounds $\limsup _{t \rightarrow \infty}\left|\tilde{x}_{0}(t)\right| \leq 0.028, \limsup _{t \rightarrow \infty}\left|\tilde{x}_{\mathrm{dz}}(t)\right| \leq 0.022$ for $\lambda=4$, and $\lim \sup _{t \rightarrow \infty}\left|\tilde{x}_{\mathrm{dz}}(t)\right| \leq 0.016$ for $\lambda=2$, showing an improvement of the steady-state performances also in the presence of high-frequency measurement noise, as originally pointed out in [23], [24].

\section{User Guidelines for Dead-Zone Parameter Tuning}

As in Section III, the bounds derived in the proof of Theorem 2 for the design of parameters $\Lambda, \Theta$ may result to be very conservative, and their tuning based on experimental simulations can be more effective, as also illustrated in the previous example.

For the tuning of the parameters, one should first select values of $\Lambda$ of the same order of magnitude as the decay rate of the estimation error of the original observer in order not the deteriorate too much the transient response. Once $\Lambda$ is fixed, it is suggested to increase as much as possible the ratio $\Theta / \Lambda$ to reduce the DC gain, and therefore improve the sensitivity to measurement noise.

\section{Mixed Redesign}

An appealing feature of the parallel developments of Sections III and IV is that the corresponding redesigns preserve Properties 1 and 2. Due to this fact, it is immediate to apply a mixed stubborn dead-zone redesign by nesting the two approaches as follows 5

$$
\begin{aligned}
\dot{z} & =\varphi(z, u)+G \kappa\left(z, \operatorname{sat}_{\sigma_{S}}\left(\mathrm{dz}_{\sigma_{D}}(y-h(\hat{x}))\right)\right), \\
\dot{\sigma}_{S} & =-\Lambda_{S} \sigma_{S}+\Theta_{S}|y-h(\hat{x})|_{\mathrm{cw}}, \\
\dot{\sigma}_{D} & =-\Lambda_{D} \sigma_{D}+\Theta_{D}|y-h(\hat{x})|_{\mathrm{cw}}, \\
\hat{x} & =\psi(z),
\end{aligned}
$$

where $\left(z, \sigma_{S}, \sigma_{D}\right) \in \mathbb{R}^{\varrho} \times \mathbb{R}_{>0}^{m} \times \mathbb{R}_{>0}^{m}$ is the redesigned observer state and $\Lambda_{S}:=\operatorname{diag}\left(\lambda_{S 1}, \ldots, \lambda_{S m}\right)>0, \Theta_{S}:=$ $\operatorname{diag}\left(\theta_{S 1}, \ldots, \theta_{S m}\right)>0, \Lambda_{D}:=\operatorname{diag}\left(\lambda_{D 1}, \ldots, \lambda_{D m}\right)>0$, $\Theta_{D}:=\operatorname{diag}\left(\theta_{D 1}, \ldots, \theta_{D m}\right) \geq 0$, are design parameters. The mixed redesign allows achieving both the desirable features of the saturation and dead-zone redesigns, discussed in the previous sections, while preserving the ISS-properties of the original observer (4), as stated by the following theorem, whose proof is omitted because it is an immediate consequence of Theorems 1 and 2 .

Theorem 3 Consider system (3) and suppose Assumption 1 holds. Suppose observer (4) is an ISS observer enjoying Properties 1 and 2. Then, there exist choices of $\Lambda_{S}, \Lambda_{D}, \Theta_{S}, \Theta_{D}$ such that observer (54) is an ISS observer for system (3) enjoying Properties 1 and 2.

\section{Redesign of Relevant Classes of Estimators}

In this section we show that a number of observer architectures satisfy Properties 1, 2, namely it is possible to apply the stubborn and dead-zone redesigns of Sections III-V to these classes of estimators.

\section{A. Linear Luenberger Observers}

Consider a linear system of the form

$$
\dot{x}=A x+B u+w, \quad y=C x+v
$$

with $x \in \mathbb{R}^{n}, u \in \mathbb{R}^{p}, y \in \mathbb{R}^{m}$, and a detectable pair $(A, C)$. Under Assumption 1, consider the Luenberger observer [36]:

$$
\dot{\hat{x}}=A \hat{x}+B u+L(y-C \hat{x})
$$

where $\hat{x} \in \mathbb{R}^{n}$ is the state and $L \in \mathbb{R}^{n \times m}$ is a matrix to be selected. With respect to (4), we have $z=\hat{x}, \varrho=\rho=n, \kappa(\hat{x}, y-h(\hat{x}))=$ $L(y-C \hat{x}), G=I, \psi(\hat{x})=\hat{x}$. Following Section II and owing to the specific selection of $\kappa$, the perturbed-injection version of observer (56) corresponds to

$$
\dot{\hat{x}}=A \hat{x}+B u+L(y-C \hat{x})+d .
$$

We have the following result.

Proposition 4 Let Assumption 1 hold with $\mathcal{X}_{0}=\mathcal{X}=\mathbb{R}^{n}, \mathcal{U} \in \mathbb{R}^{p}$, and $\mathcal{W}$ being any arbitrarily large compact subset of $\mathbb{R}^{n}$. Let $L$ be chosen such that $A-L C$ is Hurwitz. Then, Properties 1-2 are satisfied by system (55) and observer (56) with the choices in Table I, $\mathcal{Z}=\mathbb{R}^{n}$ for any arbitrarily large compact sets $\mathcal{D} \subset \mathbb{R}^{\rho}, \mathcal{V} \subset \mathbb{R}^{m}$.

Proof: First of all, inequality (6) is automatically satisfied since $\kappa$ is linear in $y-h(\hat{x})$ and $L$ is constant. Then, consider the function

\footnotetext{
${ }^{5}$ Evidently, we can also select $\kappa\left(z, \mathrm{dz}_{\sigma_{S}}\left(\operatorname{sat}_{\sigma_{D}}(y-h(\hat{x}))\right)\right)$ in (54).
} 


\begin{tabular}{lll}
\hline$V(x, \hat{x})=\sqrt{(x-\hat{x})^{\top} P(x-\hat{x})}$ & $\psi^{-R}(x)=x$ \\
$P(A-L C)+(A-L C)^{\top} P=-2 I$ & $\underline{\alpha}(|s|)=\sqrt{\underline{\lambda}_{P}}|s|$ \\
$\bar{\kappa}=|L|$ & $c=1 / \bar{\lambda}_{P}$ & $\bar{\alpha}(|s|)=\sqrt{\bar{\lambda}_{P}}|s|$ \\
$c_{w}=\bar{\lambda}_{P} / \sqrt{\underline{\lambda}_{P}}$ & $c_{d}=\bar{\lambda}_{P} / \sqrt{\underline{\lambda}_{P}}$ & $c_{v}=|L| \bar{\lambda}_{P} / \sqrt{\underline{\lambda}_{P}}$ \\
\hline$\ell_{0}=|C| / \sqrt{\underline{\lambda}_{P}}$ & $\ell_{1}=|C(A-L C)| / \sqrt{\underline{\lambda}_{P}}$ \\
$\ell_{v}=|C L|$ & $\ell_{w}=|C|$ & $\ell_{d}=|C|$ \\
\hline
\end{tabular}

TABLE I: System and design parameters for the linear system (55) and the Luenberger observer (56).

$V$ defined in Table I with $P \in \mathbb{R}^{n \times n}$ and $P>0$. Since $\underline{\lambda}_{P} I \leq$ $P \leq \bar{\lambda}_{P} I$, we have

$$
\sqrt{\underline{\lambda}_{P}}|x-\hat{x}| \leq V(x, \hat{x}) \leq \sqrt{\bar{\lambda}_{P}}|x-\hat{x}| .
$$

From (58), we get the property of $\underline{\alpha}$ and $\bar{\alpha}$ satisfying (7), as in Table I. Define $e:=x-\hat{x}$. By differentiating along (55) and (57), we obtain

$$
\dot{e}=(A-L C) e+w-L v-d .
$$

Therefore, by differentiating $V$ along (59), and proceeding as in [32, p. 203], the Dini derivative of $V$ is such that

$$
\begin{aligned}
D^{+} V & \leq-\frac{|e|^{2}}{\sqrt{e^{\top} P e}}+\frac{1}{\sqrt{e^{\top} P e}} e^{\top} P(w-L v-d) \\
& \leq-c V+c_{w}|w|+c_{v}|v|+c_{d}|d|,
\end{aligned}
$$

where $c, c_{w}, c_{d}, c_{v}$ are selected as in Table I. Note that, by linearity, the sets $\mathcal{V}, \mathcal{D}$ can be selected arbitrarily large. This shows that (8) in Property 1 holds. To show (9), we have from (58), $|C(x-\hat{x})| \leq$ $|C||x-\hat{x}| \leq \ell_{0} V(x, \hat{x})$, with $\ell_{0}$ defined in Table I, which proves (9a) in Property 2. Finally, from (58) and (59) we obtain

$$
\begin{aligned}
\left|D^{+} C(x-\hat{x})\right| & =\left|D^{+} C e\right| \\
& =|C(A-L C) e+C w-C L v+C d| \\
& \leq \ell_{1} V(x, \hat{x})+\ell_{v}|v|+\ell_{w}|w|+\ell_{d}|d|,
\end{aligned}
$$

with $\ell_{1}, \ell_{v}, \ell_{w}, \ell_{d}$ defined in Table I. This shows that (9b) holds and concludes the proof.

In view of Proposition 4, we can apply the stubborn redesign (10) or the dead-zone redesign (40) to filter (56) as follows

$$
\begin{aligned}
& \dot{\hat{x}}=A \hat{x}+B u+L \Phi_{\sigma}(y-C \hat{x}) \\
& \dot{\sigma}=-\Lambda \sigma+\Theta|y-C \hat{x}|_{\mathrm{cw}}
\end{aligned}
$$

where $\Phi_{\sigma}=$ sat $_{\sigma}$ or $\Phi_{\sigma}=\mathrm{dz}_{\sigma}$, according to the desired design, and for some diagonal $\Lambda, \Theta>0$.

Remark 1 The redesign (61) can be seen as an alternative to the designs proposed in [6], [24] having a few desirable enhancements. First, in [6] a single saturation threshold is used for all the input channels, whereas here we adopt different values $\sigma_{i}$ of the saturation levels. This anticipates improved transients in multi-output systems, especially when outliers affect only some of the outputs at hand. Secondly, the dynamics of the saturation threshold $\sigma$ used in [6], [24] is quadratic in the output error, whereas we use here a linear injection depending on the norm of the output error.

\section{B. Observers for Lipschitz Systems}

Consider a system of the form

$$
\dot{x}=A x+\phi(x, u)+w, \quad y=C x+v
$$

where the pair $(C, A)$ is detectable and $\phi(\cdot, u)$ is a globally Lipschitz function uniformly in $u$, i.e.,

$$
|\phi(x, u)-\phi(\hat{x}, u)| \leq k_{\phi}|x-\hat{x}|, \quad \forall x, \hat{x} \in \mathcal{X}, u \in \mathcal{U},
$$

for some scalar $k_{\phi}>0$. We suppose that Assumption 1 holds and we consider an observer of the form

$$
\dot{\hat{x}}=A \hat{x}+\phi(\hat{x}, u)+L(y-C \hat{x}),
$$

where $\hat{x} \in \mathbb{R}^{n}$ is the state and $L \in \mathbb{R}^{n \times m}$ is a matrix to be selected. With respect to (4), we have $z=\hat{x}, \rho=\varrho=n, \kappa(\hat{x}, y-h(\hat{x}))=$ $L(y-C \hat{x}), G=I, \psi(\hat{x})=\hat{x}$ for any $\hat{x} \in \mathbb{R}^{n}$. As in Section VIA, we can derive the perturbed-injection version of observer (64), which is omitted, here and in the following, for space reasons. In the next proposition we follow the design proposed in [37], [45] and establish the applicability of the saturation and dead-zone redesigns to this class of systems.

Proposition 5 Let Assumption 1 hold for some sets $\mathcal{X}_{0} \subseteq \mathcal{X} \subseteq \mathbb{R}^{n}$, $\mathcal{U} \in \mathbb{R}^{p}$ for which (63) holds, and for any arbitrarily large compact set $\mathcal{W} \subset \mathbb{R}^{n}$. Suppose that there exist $P=P^{\top}>0, \nu>0, \varepsilon>0$, and $Y \in \mathbb{R}^{n \times m}$ satisfying the LMI

$$
\left(\begin{array}{cc}
A^{\top} P+P A-Y C-C^{\top} Y^{\top}+\left(\nu k_{\phi}^{2}+2 \varepsilon\right) I & P \\
P & -\nu I
\end{array}\right) \leq 0,
$$

and select $L:=P^{-1} Y$. Then, Properties 1-2 are satisfied for system (62) and observer (64) with the choices in Table II and $\mathcal{Z}=\mathbb{R}^{n}$ for any arbitrarily large compact sets $\mathcal{D} \subset \mathbb{R}^{\rho}, \mathcal{V} \subset \mathbb{R}^{m}$.

Proof: As in the proof of Proposition 4, the function $V$ defined in Table II satisfies (58). Let us consider the Dini derivative of $V^{2}(x, \hat{x})$ as in Section VI-A, i.e.,

$$
\begin{aligned}
D^{+}\left(e^{\top} P e\right)= & e^{\top}\left((A-L C)^{\top} P+P(A-L C)\right) e \\
& +2 e^{\top} P(\phi(x, u)-\phi(\hat{x}, u))
\end{aligned}
$$

and use Young's inequality and bound (63) to get

$$
\begin{aligned}
& 2 e^{\top} P(\phi(x, u)-\phi(\hat{x}, u)) \\
& \leq \frac{1}{\nu} e^{\top} P P e+\nu|\phi(x, u)-\phi(\hat{x}, u)|^{2} \leq \frac{1}{\nu} e^{\top} P P e+\nu k_{\phi}^{2} e^{\top} I e .
\end{aligned}
$$

Applying a Schur complement to inequality (65), we obtain

$$
(A-L C)^{\top} P+P(A-L C)+\frac{1}{\nu} P P+\nu k_{\phi}^{2} I \leq-2 \varepsilon I,
$$

for some $\varepsilon>0$. Using (63) and following the proof of Proposition 4, we get

$$
D^{+} V \leq-\frac{\varepsilon|e|^{2}}{\sqrt{e^{\top} P e}}+\frac{1}{\sqrt{e^{\top} P e}} e^{\top} P(w-L v-d),
$$

showing (8) with $c, c_{v}, c_{w}, c_{d}$ defined in Table II. The rest of the proof follows the same arguments as those of the proof of Proposition 4.

In view of Proposition 5, we can apply the stubborn redesign (10) or the dead-zone redesign (40) to filter (64) as follows

$$
\begin{aligned}
& \dot{\hat{x}}=A \hat{x}+\phi(\hat{x}, u)+L \Phi_{\sigma}(y-C \hat{x}) \\
& \dot{\sigma}=-\Lambda \sigma+\Theta|y-C \hat{x}|_{\mathrm{cw}}
\end{aligned}
$$

for some diagonal $\Lambda, \Theta>0$ and $\Phi_{\sigma}=\operatorname{sat}_{\sigma}$ or $\Phi_{\sigma}=\mathrm{dz}_{\sigma}$.

Remark 2 For nonlinear systems of the form

$$
\dot{x}=A x+\Gamma \mathfrak{g}(H x)+\mathfrak{r}(y, u)+w, \quad y=C x+v,
$$

the following observer, based on the circle-criterion, is proposed in [8]

$$
\dot{\hat{x}}=A \hat{x}+L(y-C \hat{x})+\Gamma \mathfrak{g}(H \hat{x}+K(y-C \hat{x}))+\mathfrak{r}(y, u) .
$$

This observer can be expressed as in (3) as follows

$$
\dot{\hat{x}}=A \hat{x}+\Gamma \mathfrak{g}(H \hat{x})+\mathfrak{r}(y, u)+\kappa(\hat{x}, y-h(\hat{x}))
$$




\begin{tabular}{lll}
\hline$V(x, \hat{x})=\sqrt{(x-\hat{x})^{\top} P(x-\hat{x})}$ & $\psi^{-R}(x)=x$ \\
$\underline{\alpha}(|s|)=\sqrt{\underline{\lambda}_{P}}|s|$ & $\bar{\alpha}(|s|)=\sqrt{\bar{\lambda}_{P}}|s|$ & $\bar{\kappa}=|L|$ \\
$c=\varepsilon / \bar{\lambda}_{P}$ & $c_{w}=c_{d}=\bar{\lambda}_{P} / \sqrt{\underline{\lambda}_{P}}$ & $c_{v}=|L| \bar{\lambda}_{P} / \sqrt{\underline{\lambda}_{P}}$ \\
\hline$\ell_{0}=|C| / \sqrt{\underline{\lambda}_{P}}$ & $\ell_{1}=|C(A-L C)| / \sqrt{\underline{\lambda}_{P}}$ \\
$\ell_{v}=|C L|$ & $\ell_{w}=|C|$ & $\ell_{d}=|C|$ \\
\hline
\end{tabular}

TABLE II: System and design parameters for the Lipschitz nonlinear system (62) and the nonlinear observer (64).

with $\kappa(\hat{x}, y-h(\hat{x})):=L(y-C \hat{x})+\Gamma[\mathfrak{g}(H \hat{x}+K(y-C \hat{x}))-$ $\mathfrak{g}(H \hat{x})]$. In [8], it is shown that the gains $L, K$ can be selected to ensure the ISS of the estimation error. Therefore, as in Proposition 5, Properties 1-2 follow from using the square root of the quadratic Lyapunov function presented in [8].

\section{Observers for Input-affine Systems}

Consider a system of the form

$$
\dot{x}=A(u) x+B(u)+w, \quad y=C x+v
$$

where $u \mapsto A(u) \in \mathbb{R}^{n \times n}$ and $u \mapsto B(u) \in \mathbb{R}^{n}$ are continuous. We suppose that Assumption 1 holds. For this class of systems, an observer was originally proposed in [29]. We consider the design approach presented in [17] for estimators of the form

$$
\dot{\hat{x}}=A(u) \hat{x}+B(u)+P^{-1} C^{\top}(y-C \hat{x}),
$$

where $\hat{x} \in \mathbb{R}^{n}$ is the state, $u$ is a known input function, and $t \mapsto$ $P(t)=P(t)^{\top} \in \mathbb{R}^{n \times n}$ is the solution of

$$
\dot{P}=-2 \mu P-A(u)^{\top} P-P A(u)+2 C^{\top} C,
$$

with $P(0)>0$ and $\mu>0$. With respect to (4), we have $\rho=n$ and

$$
\begin{array}{ll}
z=(\hat{x}, \operatorname{vec}(P)), & \kappa(z, y-h(\hat{x}))=P^{-1} C^{\top}(y-C \hat{x}), \\
\varrho=n+n(n+1) / 2 & \psi(z)=\hat{x}, \quad G=\left[\begin{array}{ll}
I & 0
\end{array}\right]^{\top} .
\end{array}
$$

First, we recall the following in [17, Lemma 2.1, p. 177].

Lemma 2 Suppose that the input $t \mapsto u(t)$ is regularly persistent (see [17, Definition 2.1]). Then there exists $\mu^{\star}>0$ such that, for any $\mu \geq \mu^{\star}$, the solution $t \mapsto P(t)$ to (68b) satisfies $\underline{p} I \leq P(t) \leq \bar{p} I$ for all $t \geq 0$ and for some $\underline{p}, \bar{p} \in \mathbb{R}$ such that $0<\underline{p}<\bar{p}$.

Based on the results in [17], we establish below the applicability of the proposed redesigns to observer (68).

Proposition 6 If input $t \mapsto u(t)$ is regularly persistent (see [17, Definition 2.1]), then Properties 1-2 are satisfied for system (67) and observer (68) with the choices in Table III.

Proof: First of all, note that, in view of the property of $\psi^{-R}$ in Table III, we have $|x-\hat{x}| \leq \mid(x, 0)-(\hat{x}, \operatorname{vec}(P)))|=| \psi^{-R}(x)-z \mid$. Therefore, by using the previous inequality and Lemma 2 , it follows that $\sqrt{p}|x-\hat{x}| \leq V(x, z) \leq \sqrt{\bar{p}}\left|\psi^{-R}(x)-z\right|$, by which we obtain bound (58) with $\underline{\alpha}, \bar{\alpha}$ given in Table III. Now, let us write the perturbed-injection dynamics of the estimation error according to (5), which, using Table III, is

$$
\dot{e}=\left(A(u)-P^{-1} C^{\top} C\right) e+w-P^{-1} C^{\top} v-d .
$$

Let us now consider the Dini derivative of $V^{2}(x, \hat{x})$. It reads

$$
D^{+}\left(e^{\top} P e\right)=-2 \mu e^{\top} P e+2 e^{\top} P(w-d)+2 e^{\top} C^{\top} v
$$

\begin{tabular}{lll}
\hline$V(x, z)=\sqrt{(x-\hat{x})^{\top} P(x-\hat{x})}$ & $\psi^{-R}(x)=(x, 0)$, \\
$\underline{\alpha}(|s|)=\sqrt{\underline{p}}|s|$ & $\bar{\alpha}(|s|)=\sqrt{\bar{p}}|s|$ & $\bar{\kappa}=|C| / \bar{p}$ \\
$c=\mu$ & $c_{w}=c_{d}=\bar{p} / \sqrt{\underline{p}}$ & $c_{v}=|C| \overline{\sqrt{p}}$ \\
\hline$\ell_{0}=|C| / \sqrt{\underline{\lambda}}$ & $\ell_{1}=|C|\left(|A|+|C|^{2} / \underline{p}\right) / \sqrt{p}$ \\
$\ell_{v}=|C|^{2} / \underline{p}$ & $\ell_{w}=|C|$ & $\ell_{d}=|C|$ \\
\hline
\end{tabular}

TABLE III: System and design parameters for the input-affine system (67) and observer (68).

and, by means of $\left|e^{\top} / \sqrt{e^{\top} P e}\right| \leq 1 / \sqrt{\underline{p}}$ holding for all $e \in \mathbb{R}^{n} \backslash\{0\}$ and $P$ satisfying Lemma 2 , we obtain

$$
D^{+} V=-\mu V+\frac{1}{\sqrt{e^{\top} P e}} e^{\top}\left(P(w-d)+C^{\top} v\right)
$$

from which we obtain (8) with $c_{w}, c_{d}, c_{v}$ defined in Table III. The rest of the proof follows the same arguments as those of the proof of Proposition 4.

In view of Proposition 6, we can apply the stubborn (10) or deadzone (40) redesigns to filter (68) as follows

$$
\begin{aligned}
\dot{\hat{x}} & =A(u) \hat{x}+B(u)+P^{-1} C^{\top} \Phi_{\sigma}(y-C \hat{x}) \\
\dot{P} & =-2 \mu P-A(u)^{\top} P-P A(u)+2 C^{\top} C \\
\dot{\sigma} & =-\Lambda \sigma+\Theta|y-C \hat{x}|_{\mathrm{cW}},
\end{aligned}
$$

for some diagonal $\Lambda, \Theta>0$ and $\Phi_{\sigma}=\operatorname{sat}_{\sigma}$ or $\Phi_{\sigma}=\mathrm{dz}_{\sigma}$.

\section{Extended Kalman Filters}

Consider a system of the form

$$
\dot{x}=f(x, u)+w, \quad y=h(x)+v
$$

where $x \in \mathbb{R}^{n}$ is the state, $u \in \mathbb{R}^{p}$ is a known input, and $y \in \mathbb{R}^{m}$ is the measured output. Suppose that Assumption 1 holds for some given compact sets $\mathcal{X} \subset \mathbb{R}^{n}, \mathcal{U} \subset \mathbb{R}^{p}$ and for some (small enough) compact set $\mathcal{W} \subset \mathbb{R}^{n}$. Also suppose that functions $f, h$ are $C^{1}$. Given some $\varepsilon>0$, assume that there exist $k_{h}, k_{f}, k_{h f}>0$ such that the following inequalities hold

$$
\begin{aligned}
|h(x)-h(\hat{x})| & \leq k_{h}|x-\hat{x}| \\
|f(x, u)-f(\hat{x}, u)| & \leq k_{f}|x-\hat{x}| \\
\left|\left(\frac{\partial h}{\partial x}(x)-\frac{\partial h}{\partial x}(\hat{x})\right) f(x, u)\right| & \leq k_{h f}|x-\hat{x}|
\end{aligned}
$$

for all $x \in \mathcal{X}$, all $\hat{x} \in \mathbb{R}^{n}$ such that $|x-\hat{x}| \leq \varepsilon$, and all $u \in \mathcal{U}$.

We consider the EKF with prescribed degree of stability proposed in [38], i.e.,

$$
\begin{aligned}
& \dot{\hat{x}}=f(\hat{x}, u)+K(\hat{x})(y-h(\hat{x})) \\
& K(\hat{x})=P C(\hat{x})^{\top} R^{-1}
\end{aligned}
$$

with $t \mapsto P(t)$ solution to the Riccati differential equation

$$
\begin{array}{r}
\dot{P}=(A(\hat{x}, u)+\mu I) P+P\left(A(\hat{x}, u)^{\top}+\mu I\right) \\
-P C(\hat{x})^{\top} R^{-1} C(\hat{x}) P+Q, \\
A(\hat{x}, u):=\frac{\partial f}{\partial x}(\hat{x}, u), \quad C(\hat{x}):=\frac{\partial h}{\partial x}(\hat{x}),
\end{array}
$$

$\mu \geq 0, R=R^{\top}>0$, and $Q=Q^{\top}>0$. Note that we recover the form of a standard EKF (see, e.g., [19], [34]) by selecting $\mu=0$ in (72c). With respect to the compact notation (4), we have $\rho=n$,

$$
\begin{array}{ll}
z=(\hat{x}, \operatorname{vec}(P)), & \kappa(z, y-h(\hat{x}))=K(\hat{x})(y-h(\hat{x})), \\
\varrho=n+n(n+1) / 2 & \psi(z)=\hat{x}, \quad G=\left[\begin{array}{ll}
I & 0
\end{array}\right]^{\top} .
\end{array}
$$




\begin{tabular}{lll}
\hline $\mathcal{W} \subset \mathbb{B}_{\delta}$ & $\mathcal{V} \subset \mathbb{B}_{\delta}$ & $\mathcal{D} \subset \mathbb{B}_{\varepsilon}$ \\
\hline$V(x, z)=\sqrt{W(x, z)}$ & $\psi^{-R}(x)=(x, 0)$ & \\
$\underline{\alpha}(|s|)=|s| / \sqrt{\bar{p}}$ & $\bar{\alpha}(|s|)=|s| / \sqrt{p}$ & $\bar{\kappa}=\bar{p} \bar{c}\left|R^{-1}\right|$ \\
$c=\mu+\underline{\lambda}_{Q} \underline{p} /\left(4 \bar{p}^{2}\right)$ & $c_{w}=c_{d}=\sqrt{\bar{p}} / \underline{p}$ & $c_{v}=\bar{\kappa} \sqrt{\bar{p}} / \underline{p}$ \\
\hline$\ell_{0}=k_{h} / \sqrt{p}$ & $\ell_{1}=\left(k_{h f}+\bar{c} k_{f}+\bar{c} \bar{\kappa} k_{h}\right) / \sqrt{p}$ \\
$\ell_{v}=\bar{c} \bar{\kappa}$ & $\ell_{w}=\bar{c}$ & $\ell_{d}=\bar{c}$ \\
\hline
\end{tabular}

TABLE IV: System and design parameters for system (70) and the EKF (72).

and $\varphi$ defined according to dynamics (72a), (72c). Likewise in [38], we define the functions (obtained via expansions of $f, h$ into power series)

$$
\begin{aligned}
\phi(x, \hat{x}, u) & :=f(x, u)-f(\hat{x}, u)-A(\hat{x}, u)(x-\hat{x}), \\
\chi(x, \hat{x}) & :=h(x)-h(\hat{x})-C(\hat{x})(x-\hat{x}) .
\end{aligned}
$$

Following [38], the next assumption is stated.

Assumption 2 (Assumptions 1-3 in [38]) There exist $\varepsilon_{\phi}$, $\varepsilon_{\chi}$, $k_{\phi}, k_{\chi}, \bar{c}, \bar{p}, \underline{p}>0$ such that

1) $|\phi(x, \hat{x}, u)| \leq k_{\phi}|x-\hat{x}|^{2}$ for all $u \in \mathbb{R}^{p}$ and for all $x, \hat{x} \in \mathbb{R}^{n}$ such that $|x-\hat{x}| \leq \varepsilon_{\phi}$

2) $|\chi(x, \hat{x})| \leq k_{\chi}|x-\hat{x}|^{2}$ for all $x, \hat{x} \in \mathbb{R}^{n}$ such that $|x-\hat{x}| \leq$ $\varepsilon_{\chi}$

3) $|C(x(t))| \leq \bar{c}$ and $|C(\hat{x}(t))| \leq \bar{c}$ for any solution to (70), (72);

4) the solution $t \mapsto P(t)$ to (72c) satisfies $\underline{p} I \leq P(t) \leq \bar{p} I$ for any solution to (70), (72).

Under the previous conditions, the following result ensuring the (local) stability of the estimation error can be proved.

Proposition 7 (Main Theorem in [38]) There exist $\varepsilon_{0}, \beta_{0}>0$ and $\bar{\mu}>\mu \geq 0$ such that solutions to system (70) and estimator (72), with $w=0$, satisfy

$$
|x(t)-\hat{x}(t)| \leq \beta_{0} \exp (-\bar{\mu} t)|x(0)-\hat{x}(0)|
$$

for any initial condition $x, \hat{x} \in \mathbb{R}^{n}$ satisfying $|x-\hat{x}| \leq \varepsilon_{0}$. Moreover, this can be established with the Lyapunov function

$$
W(x, z):=(x-\hat{x})^{\top} P^{-1}(x-\hat{x}) .
$$

Based on the above setup, mostly inspired by [38], we have the following result.

Proposition 8 Under Assumption 2, there exists $\varepsilon_{1}, \delta>0$ such that Properties 1-2 are satisfied for system (70) and estimator (72) with the choices in Table IV and for any initial condition $x, \hat{x} \in \mathbb{R}^{n}$ satisfying $|x-\hat{x}| \leq \varepsilon_{1}$ and disturbances $v \in \mathbb{R}^{m}, w \in \mathbb{R}^{n}$ such that $|v| \leq \delta$ and $|w| \leq \delta$.

Proof: First of all, consider system (70) and observer (72) when $w=0, v=0$, and $d=0$, and suppose that the inequalities in Assumption 2 are verified, namely $|x-\hat{x}| \leq \min \left\{\varepsilon_{\phi}, \varepsilon_{\chi}\right\}$. By following the computations in [38], we have that the Lyapunov function $W$ defined in (74), satisfies $\dot{W} \leq-\left(2 \mu+\frac{\lambda_{Q} \underline{p}}{2 \bar{p}^{2}}\right) W$. As a consequence, when considering the time derivative of $V$ defined in Table IV, we easily obtain

$$
\dot{V} \leq-\left(\mu+\frac{\underline{\lambda}_{Q} \underline{p}}{4 \bar{p}^{2}}\right) V=-c V
$$

with $c$ defined in Table IV. Since $t \mapsto P(t)$ is uniformly bounded by item 4) of Assumption 2, (75) proves local exponential stability of the estimation error to zero. Recall that local exponential stability implies local ISS, see [32]. As a consequence, in order to show (8), we first recall the following inequalities

$$
\begin{array}{ll}
\frac{1}{\sqrt{\bar{p}}}|x-\hat{x}| \leq V(x, z) \leq \frac{1}{\sqrt{p}}|x-\hat{x}|, \quad & \forall x, \hat{x} \in \mathbb{R}^{n}, \\
\frac{\left|(x-\hat{x})^{\top} P^{-1}\right|}{\sqrt{(x-\hat{x})^{\top} P^{-1}(x-\hat{x})}} \leq \frac{\sqrt{\bar{p}}}{\underline{p}}, & \forall x \neq \hat{x},
\end{array}
$$

where $t \mapsto P(t)$ satisfies item 4) of Assumption 2. From inequalities (75) and (76), we can derive (8) with $c_{w}, c_{d}, c_{v}$ selected as in Table IV. Finlly, since (75) holds only for $|x-\hat{x}| \leq \varepsilon_{0}$, for any $\varepsilon_{1} \in\left(0, \varepsilon_{0}\right]$, then there exists $\delta \in[0, \infty)$ such that (8) holds for all $|x-\hat{x}| \leq \varepsilon_{1},|w| \leq \delta,|d| \leq \delta$ and $|v| \leq \delta$, thus proving Property 1 .

Now, by combining (71) and (76), inequality (9a) is verified with $\ell_{0}$ in Table IV. Finally, to verify (9b), compute

$$
\begin{aligned}
& \mid D^{+}(h(x)-h(\hat{x})) \mid \leq \\
& \leq \mid \frac{\partial h}{\partial x}(x)(f(\hat{x}, u)+w) \\
& \quad \quad-\frac{\partial h}{\partial x}(\hat{x})(f(x, u)+K(\hat{x})(h(x)+v-h(\hat{x}))+d) \mid \\
& \leq \mid\left.\frac{\partial h}{\partial x}(x)-\frac{\partial h}{\partial x}(\hat{x})\right) f(x, u) \mid \\
& \quad+\left|\frac{\partial h}{\partial x}(\hat{x})(f(x, u)-f(\hat{x}, u))\right| \quad+\left|\frac{\partial h}{\partial x}(\hat{x}) K(\hat{x})(h(x)-h(\hat{x}))\right| \\
& \quad+\left|\frac{\partial h}{\partial x}(x) w\right|+\left|\frac{\partial h}{\partial x}(\hat{x}) d\right|+\left|\frac{\partial h}{\partial x}(\hat{x}) K(\hat{x}) v\right| \\
& \leq\left(k_{h f}+\bar{c} k_{f}+\bar{c} \bar{\kappa} k_{h}\right)|x-\hat{x}|+\bar{c}|w|+\bar{c}|d|+\bar{c} \bar{\kappa}|v|,
\end{aligned}
$$

which holds for all $x \in \mathcal{X}$ and all $\hat{x} \in \mathbb{R}^{n}$ such that $|x-\hat{x}| \leq \varepsilon_{1}$ and for all $u \in \mathcal{U}$. The proof is completed by selecting $\ell_{1}, \ell_{w}, \ell_{v}$, $\ell_{d}$ as in Table IV.

Based on the results above, the stubborn redesign (10) or the deadzone redesign (40) of the EKF correspond to

$$
\begin{aligned}
\dot{\hat{x}} & =f(\hat{x}, u)+K(\hat{x}) \Phi_{\sigma}(y-h(\hat{x})) \\
\dot{\sigma} & =-\Lambda \sigma+\Theta|y-h(\hat{x})|_{\mathrm{cw}}
\end{aligned}
$$

for some diagonal $\Lambda, \Theta>0$ together with the corresponding selections (72b) and (72c), and $\Phi_{\sigma}=$ sat $_{\sigma}$ or $\Phi_{\sigma}=\mathrm{dz}_{\sigma}$.

Remark 3 Similar conditions can be derived for a linear systems and a Kalman observer by combining the results of this section and those of linear observers. Indeed, this section generalizes [26], where the authors, inspired by [6], propose a stubborn redesign for the Kalman filter.

\section{E. Observers for Systems in Canonical Observability Form}

Consider the class of single-output nonlinear systems

$$
\dot{x}=A_{n} x+B_{n} \phi(x, u)+w, \quad y=C_{n} x+v
$$

where $\left(A_{n}, B_{n}, C_{n}\right)$ is a triplet in prime form ${ }^{6}$ of dimension $n$ and $\phi$ is a locally Lipschitz function. Also suppose that Assumption 1 holds, with $\mathcal{X}, \mathcal{W}, \mathcal{U}$ being some given compact sets. For system (3), a typical approach is that of high-gain observers [33]. For this class of estimators, the use of dynamic saturations and dead-zones has been already studied in [9], [24]. Therefore, in this section, we focus on

${ }^{6}$ A triplet of matrices $(A, B, C)$ is said to be in prime form when $A$ is a shift matrix (all 1's on the upper diagonal and all 0 's elsewhere), $B^{\top}=$ $\left(\begin{array}{lll}0 \cdots 0 & 1\end{array}\right)$ and $C=\left(\begin{array}{lll}1 & 0 \cdots 0\end{array}\right)$. 
the low-power high-gain observer introduced in [11], for which the analysis is more involved. In particular, consider

$$
\begin{gathered}
\dot{z}_{1}=A_{2} z_{1}+N z_{2}+D_{2}(g) K_{1}\left(y-C_{2} z_{1}\right), \\
\dot{z}_{i}=A_{2} z_{i}+N z_{i+1}+D_{2}(g) K_{i}\left(B_{2}^{\top} z_{i-1}-C_{2} z_{i}\right), \\
i=2, \ldots, n-2 \\
\dot{z}_{n-1}=A_{2} z_{n-1}+B_{2} \phi_{s}(\hat{x}, u) \\
\quad+D_{2}(g) K_{n-1}\left(B_{2}^{\top} z_{n-2}-C_{2} z_{n-1}\right),
\end{gathered}
$$

where $z=\left(z_{1}, \ldots, z_{n-1}\right) \in \mathbb{R}^{2 n-2}$ is the state, $\hat{x}=\Gamma z \in \mathbb{R}^{n}$ is the estimate, with $\Gamma:=\operatorname{blkdiag}\left(C_{2}, \ldots, C_{2}, I_{2}\right) \in \mathbb{R}^{n \times(2 n-2)}$, $\left(A_{2}, B_{2}, C_{2}\right)$ is a triplet in prime form of dimension $2, N:=B_{2} B_{2}^{\top}$, $K_{i}:=\left(k_{i 1} k_{i 2}\right)^{\top}, i=1, \ldots, n-1$, are matrices to be chosen, $D_{2}(g):=\operatorname{diag}\left(g, g^{2}\right)$, where $g$ is the so-called high-gain parameter (to be chosen large enough) and $\phi_{s}$ is a globally bounded function designed by saturating $\phi$ in such a way that $\phi_{s}(x, u)=\phi(x, u)$ for all $(x, u) \in \mathcal{X} \times \mathcal{U}$. Note that, with respect to (4), we have $\rho=n$, $\varrho=2 n-2, \kappa(z, y-h(\hat{x}))=K_{1}\left(y-C_{2} z_{1}\right), G=\left[\begin{array}{llll}I & 0 & \cdots & 0\end{array}\right]^{\top}$, and $\psi(z)=\Gamma z$. Finally, in order to state the main result of this section, let the matrices $M_{i} \in \mathbb{R}^{2 i \times 2 i}, i=1, \ldots, n-1$ be recursively defined as $M_{1}:=A_{2}-K_{1} C_{2}$,

$$
M_{i}:=\left(\begin{array}{cc}
M_{i-1} & B_{2(i-1)} B_{2}^{\top} \\
K_{i} B_{2(i-1)}^{\top} & A_{2}-K_{i} C_{2}
\end{array}\right), M:=M_{n-1},
$$

for $i=2, \ldots, n-1$, where $B_{i}$ is in prime form, $\Psi_{g}:=$ $\operatorname{blkdiag}\left(\Psi_{g}^{1}, \ldots, \Psi_{g}^{n-1}\right), \Psi_{g}^{i}:=g^{2-i} D_{2}(g)^{-1}$, and

$$
\Upsilon:=\left(b_{1}, b_{2}, b_{2}, \ldots, b_{i}, b_{i}, \ldots, b_{n-1}, b_{n}\right) \in \mathbb{R}^{(2 n-2) \times n}
$$

where $b_{i}$ is a row vector of dimension $n$ with a 1 at the $i$-th entry and all the other elements being zero. Note that $\Upsilon$ is a right-inverse of $\Gamma$, namely $\Gamma \Upsilon=I$.

Proposition 9 Let $K_{i}, i=1, \ldots, n-1$ be such that matrix $M$ in (80) is Hurwitz. ${ }^{7}$ There exists $g^{\star} \geq 1$ such that, for any $g>g^{\star}$, Properties 1-2 are satisfied for system (78) and observer (79) with the choices in Table $V$.

Proof: By following [11], consider the change of coordinates $e:=\Psi_{g}(z-\Upsilon x)$, by which observer (79) with the perturbedinjection dynamics, as in (5), reads

$$
\dot{e}=g M e+B_{2 n-2} \Delta_{\phi}(e, x, u)+\Psi_{g} \Upsilon w+g G \kappa(z) v-\Psi_{g} G d
$$

where $\Delta_{\phi}(e, x, u):=g^{-(n-1)}\left(\phi_{s}\left(\Gamma \Psi_{g}^{-1} e+x, u\right)-\phi(x, u)\right)$. From the Lipschitz properties of $\phi$ and the boundedness of $\phi_{s}, \mathcal{X}$, and $\mathcal{U}$, there exists $L_{\phi}>0$ such that $\left|\Delta_{\phi}(e, x, u)\right| \leq L_{\phi}|e|$ for all $e \in \mathbb{R}^{2 n-2}, x \in \mathcal{X}, u \in \mathcal{U}$, and $g \geq 1$. In these coordinates, the function $V$ defined in Table $\mathrm{V}$, reads $V(e)=\sqrt{e^{\top} P e}$, with $P$ defined in Table V. By property of $e$, and using $g \geq 1$, it follows that $V$ satisfies

$$
\frac{\sqrt{\underline{\lambda}_{P}}}{g^{n-1}}|z-\Upsilon x| \leq \sqrt{\underline{\lambda}_{P}}|e| \leq V(e) \leq \sqrt{\bar{\lambda}_{P}}|e| \leq \sqrt{\bar{\lambda}_{P}}|z-\Upsilon x| .
$$

Moreover, by using the properties of $\Gamma$ and $\Upsilon$, we also have $|\hat{x}-x|=$ $|\Gamma z-\Gamma \Upsilon x| \leq|z-\Upsilon x|$, and therefore

$$
g^{-(n-1)} \sqrt{\underline{\lambda}_{P}}|\hat{x}-x| \leq V(e) .
$$

\footnotetext{
${ }^{7}$ This is always possible in view of Lemma 1 in [11].
}

\begin{tabular}{lll}
\hline$V(x, z)=\sqrt{(\Upsilon x-z)^{\top} \Psi_{g} P \Psi_{g}(\Upsilon x-z)}$ & $\psi^{-R}(x)=\Upsilon x$ \\
$P M+M^{\top} P=-2 I \quad c=g / \bar{\lambda}_{P}-L_{\phi} \bar{\lambda}_{P} / \sqrt{\lambda_{P}}$ & \\
$\underline{\alpha}(|s|)=\sqrt{\bar{\lambda}_{P}} g^{-(n-1)}|s|$ & $c_{w}=2 \bar{\lambda}_{P} / \sqrt{\lambda_{P}}$ & $\bar{\kappa}=\left|K_{1}\right|$ \\
$\bar{\alpha}(|s|)=\sqrt{\bar{\lambda}_{P}}|s|$ & $c_{v}=\left|K_{1}\right| \bar{\lambda}_{P} / \sqrt{\underline{\lambda}_{P}}$ & $c_{d}=\bar{\lambda}_{P} / \sqrt{\underline{\lambda}_{P}}$ \\
\hline$\ell_{0}=g^{n-1} / \sqrt{\underline{\lambda}_{P}}$ & $\ell_{1}=g^{n}\left|M_{n-1}\right| / \sqrt{\underline{\lambda}_{P}}$ & \\
$\ell_{v}=g\left|K_{1}\right|$ & $\ell_{w}=2$ & $\ell_{d}=1$ \\
\hline
\end{tabular}

TABLE V: System and design parameters for the nonlinear system (78) and the low-power high-gain observer (79).

The last inequality and bounds (82) prove (7) with $\underline{\alpha}, \bar{\alpha}, \psi^{-R}$ defined as in Table V. The Dini derivative of $V$ is such that

$$
\begin{array}{r}
D^{+} V \leq-\frac{g|e|^{2}}{\sqrt{e^{\top} P e}}+\frac{e^{\top} P}{\sqrt{e^{\top} P e}}\left(B_{2 n-2} \Delta_{\phi}(e, x, u)\right. \\
\left.+\Psi_{g} \Upsilon w+g G \kappa(z) v-\Psi_{g} G d\right)
\end{array}
$$

showing (8) with $c, c_{w}, c_{v}, c_{d}$ defined in Table V. Then, using (83), it follows that $|C(x-\hat{x})| \leq|x-\hat{x}| \leq \ell_{0} V(e)$ corresponding the first inequality in (9), with $\ell_{0}$ selected as in Table V. Finally, the second inequality in (9) is satisfied by using (81) as follows

$$
\begin{array}{r}
\left|D^{+} C(x-\hat{x})\right|=\left|D^{+} C \Gamma \Psi_{g}^{-1} e\right| \\
=\mid C \Gamma \Psi_{g}^{-1} g M_{n-1} e+C \Gamma \Psi_{g}^{-1} B_{2 n-2} \Delta_{\phi}(e, x, u) \\
\quad+C \Gamma \Upsilon w+g C \Gamma \Psi_{g}^{-1} G \kappa(z) v-C \Gamma G d \mid \\
\leq \ell_{1} V(e)+\ell_{v}|v|+\ell_{w}|w|+\ell_{d}|d|
\end{array}
$$

and by selecting $\ell_{1}, \ell_{v}, \ell_{w}, \ell_{d}$ as in Table V. This proves that Property 2 is satisfied and completes the proof.

In view of Proposition 9, we can apply the stubborn redesign (10) or the dead-zone redesign (40) by modifying (79) as follows

$$
\begin{aligned}
& \dot{z}_{1}=A_{2} z_{1}+N z_{2}+D_{2}(g) K_{1} \Phi_{\sigma}\left(y-C_{2} z_{1}\right), \\
& \dot{z}_{i}=A_{2} z_{i}+N z_{i+1}+D_{2}(g) K_{i}\left(B_{2}^{\top} z_{i-1}-C_{2} z_{i}\right) \text {, } \\
& i=2, \ldots, n-2 \\
& \dot{z}_{n-1}=A_{2} z_{n-1}+B_{2} \phi_{s}(\hat{x}, u) \\
& +D_{2}(g) K_{n-1}\left(B_{2}^{\top} z_{n-2}-C_{2} z_{n-1}\right), \\
& \dot{\sigma}=-\lambda \sigma+\theta\left|y-C_{2} z_{1}\right|
\end{aligned}
$$

for some $\lambda, \theta>0$ and $\Phi_{\sigma}=$ sat $_{\sigma}$ or $\Phi_{\sigma}=\mathrm{dz}_{\sigma}$.

Remark 4 The results of this section can be extended to the highgain observer variants in [10], [43]. For such variants, the definition of the right inverse $\psi^{-R}$ in Property 1 can be directly inherited from the Lyapunov function establishing the convergence of the observe derived in [10], [43]. Similarly, its square root can be used to establish Properties $1,2$.

Remark 5 For systems $\dot{x}=f(x, u), y=h(x)$, which are diffeomorphic to (78) via a change of coordinates $z=\Phi(x, u)$, it is possible to design an observer in the original coordinates

$$
\dot{\hat{x}}=f(\hat{x}, u)+\left(\frac{\partial \Phi}{\partial x}(\hat{x}, u)\right)^{-1} L(y-h(\hat{x})),
$$

for some choice of $L$, see, e.g., [14], [16] and references therein. Based on the ISS properties of the observer, similar arguments to those of Proposition 9 can be used to show that such observers satisfy Properties 1-2. Note however that, in this context, the invariance of a prescribed compact set $\mathcal{Z}$ may be required to prove condition (6). In this case, the redesign proposed in [14] may be a viable approach to constrain the observer state in a desired compact set. 

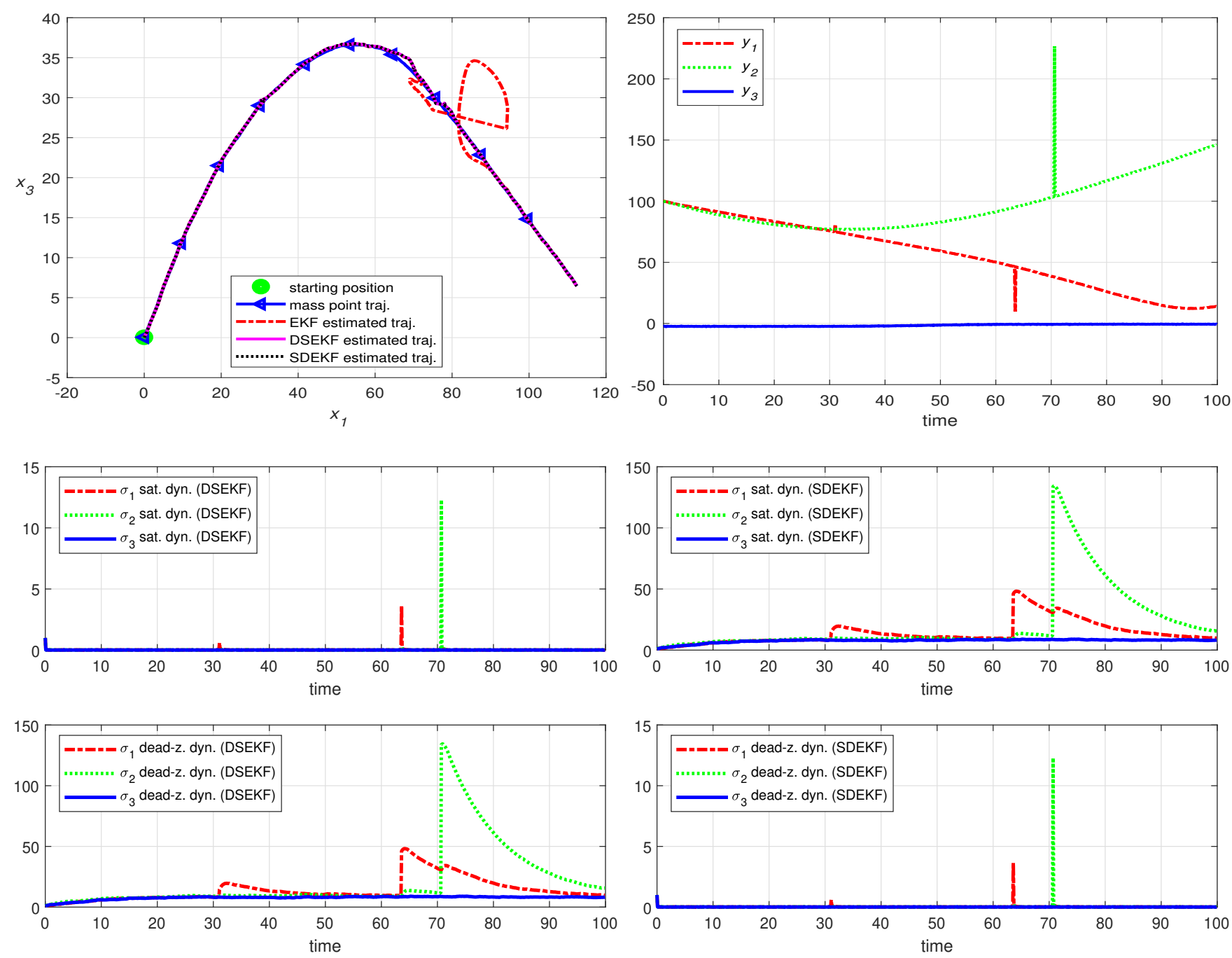

Fig. 3: EKF vs DSEKF and SDEKF in a simulation run with $r_{0}=0.01, r_{\text {out }}=100$ and a turn at half of the simulation time.

\section{Vil. Simulation Results}

In this section, we report on the simulation results obtained by applying the stubborn and dead-zone redesigns to a case study with the EKF according to Section VI-D. More specifically, we compare the standard EKF to the corresponding stubborn and dead-zone redesigns, which will be denoted by SEKF and DEKF, respectively.

Consider a fourth-order system describing the motion of a mass point with two Euclidean distance measurements and one angular distance measurement w.r.t. the points $p^{\prime}=(10,0), p^{\prime \prime}=(0,10)$, and $p^{\prime \prime \prime}=(100,100)$, respectively:

$$
\begin{aligned}
& \dot{x}_{1}=x_{2}, \dot{x}_{2}=0, \dot{x}_{3}=x_{4}, \dot{x}_{4}=0 \\
& y_{1}=\left|\left(x_{1}-p_{1}^{\prime}, x_{3}-p_{2}^{\prime}\right)\right|_{2}, y_{2}=\left|\left(x_{1}-p_{1}^{\prime \prime}, x_{3}-p_{2}^{\prime \prime}\right)\right|_{2}, \\
& y_{3}=\operatorname{atan} 2\left(x_{3}-p_{2}^{\prime \prime \prime}, x_{1}-p_{1}^{\prime \prime \prime}\right)
\end{aligned}
$$

where $\operatorname{atan} 2(\cdot, \cdot)$ denotes the four-quadrant inverse tangent function. For all the EKF-based estimators considered here, the corresponding Riccati differential equations have been solved by using a simple Euler approximation with sampling time equal to 0.01 . Specifically, we have chosen $\mu=0.1$ and the initial $P, Q$, and $R$ all equal to identity matrices $I$ of appropriate dimensions. Moreover, we have selected $\Lambda=10 I, \Theta=I$, and $\sigma(0)=(1,1,1)$ for the DEKF and $\Lambda=0.1 I, \Theta=10 I, \sigma(0)=(1,1,1)$ for the SEKF. The initial state of all the filters have been fixed equal to $(0,1,0,1)$. Different simulation setups depending on the parameters $r_{0}$ and $r_{\text {out }}$ have been analyzed: the random initialization of the plant state follows a Gaussian distribution with covariance $r_{0} I$ around $(0,1,0,1)$, while the system and measurement disturbances are taken as zero-mean Gaussian noises with covariance matrices all equal to $r_{0} I$. Measurement outliers may affect the distance measurements (i.e., $y_{1}$ and $y_{2}$ ) after a random time generated according to a uniform distribution between 0 and $1 / 3$ of the simulation time. Each outlier is obtained by amplifying the random noise of one measurement by a factor equal to $r_{\text {out }}$. Thus, a small $r_{0}$ refers to simulation runs with low levels of noises, whereas $r_{\text {out }}=1$ means that outliers do not occur in the simulations. Instead, large outliers affect the measurements with a high $r_{\text {out }}>1$. The root mean square errors (RMSEs) are reported in Tables VI and VII. The combinations of dead-zone and saturation dynamics in the stubborn dead-zone EKF (SDEKF with the saturation of the dead-zonated output error) and dead-zone stubborn EKF (DSEKF with dead-zone trimming to the saturated output error) perform quite well as compared to the EKF, as shown in Fig. 3 with the plot of a simulation run. Fig. 3 allows illustrating pictorially the excellent performances that can be achieved with the stubborn and dead-zone redesigns of Kalman filters in accordance with [5], [6], [9], where the interested reader is referred to for simulation results obtained with the redesign of Luenberger and high-gain observers. 


\begin{tabular}{|c|c|c|c|c|c|}
\cline { 2 - 6 } \multicolumn{1}{c|}{} & $r_{0}, r_{\text {out }}$ & $x_{1}$ & $x_{2}$ & $x_{3}$ & $x_{4}$ \\
\hline EKF & $0.001,1$ & 0.83589 & 0.15872 & 0.86909 & 0.15784 \\
\hline DEKF & $0.001,1$ & 0.66282 & 0.11169 & 0.69023 & 0.11082 \\
\hline EKF & $0.01,1$ & 1.2919 & 0.26095 & 1.3281 & 0.27735 \\
\hline DEKF & $0.01,1$ & 1.0887 & 0.22504 & 1.1240 & 0.24110 \\
\hline EKF & $0.1,1$ & 3.2327 & 0.40013 & 3.2396 & 0.40974 \\
\hline DEKF & $0.1,1$ & 2.8427 & 0.35844 & 2.8466 & 0.36285 \\
\hline EKF & 1,1 & 8.7003 & 0.63317 & 8.4298 & 0.61264 \\
\hline DEKF & 1,1 & 7.5973 & 0.58075 & 7.3755 & 0.56188 \\
\hline EKF & 10,1 & 24.5259 & 1.20421 & 23.2213 & 1.33571 \\
\hline DEKF & 10,1 & 18.1457 & 1.12691 & 17.5172 & 1.28463 \\
\hline
\end{tabular}

TABLE VI: RMSE medians over 1000 simulation runs for EKF and DEKF.

\begin{tabular}{|c|c|c|c|c|c|}
\cline { 2 - 6 } \multicolumn{1}{c|}{} & $r_{0}, r_{\text {out }}$ & $x_{1}$ & $x_{2}$ & $x_{3}$ & $x_{4}$ \\
\hline EKF & $0.1,1$ & 3.2327 & 0.40013 & 3.2396 & 0.40974 \\
\hline SEKF & $0.1,1$ & 3.2327 & 0.40013 & 3.2396 & 0.40974 \\
\hline EKF & $0.1,10$ & 3.6342 & 0.42489 & 3.6262 & 0.43263 \\
\hline SEKF & $0.1,10$ & 3.6342 & 0.42489 & 3.6262 & 0.43263 \\
\hline EKF & $0.1,100$ & 4.2929 & 0.47033 & 4.2659 & 0.47325 \\
\hline SEKF & $0.1,100$ & 4.0146 & 0.44724 & 3.9947 & 0.44972 \\
\hline EKF & $0.1,1000$ & 6.2936 & 0.77165 & 6.2272 & 0.72439 \\
\hline SEKF & $0.1,1000$ & 4.0969 & 0.45805 & 4.0748 & 0.45723 \\
\hline EKF & $0.1,10000$ & 16.9276 & 4.12612 & 16.0941 & 3.84125 \\
\hline SEKF & $0.1,10000$ & 4.5945 & 0.53379 & 4.5782 & 0.52979 \\
\hline
\end{tabular}

TABLE VII: RMSE medians over 1000 simulation runs for EKF and SEKF.

\section{CONCLUSION}

In this paper, two redesign approaches based on adaptive saturated and dead-zonated output error injections are investigated for a wide range of estimators with different architectures for both linear and nonlinear continuous-time systems, providing sufficient conditions that ensure preservation of a desirable ISS property from disturbances to the estimation error. The generality of the proposed redesigns is illustrated by their successful application to a large number of popular state estimators. Two new tools have been also introduced for the analysis of the effect of measurement noise of impulsive nature and constant bias. The theoretical findings are confirmed by simulation results, showing the advantages provided by our redesigns. This work opens a number of interesting future developments: the extension of the proposed approach to observers and filters for discrete-time nonlinear systems, following the preliminary directions of [6]; the development of the tools introduced in Sections III-B, IV-B to analyze the effect of measurement noise in a pure nonlinear setting by providing tighter bounds w.r.t. the classical $H_{\infty}, \mathcal{L}_{2}$ ones; the use of redesigned observers in output feedback stabilization problems; a thinner analysis of the case studies addressed in Section VI in order to obtain less restrictive ISS-gains as compared to the ones given in Tables I-V and hence more precise parameter design bounds for the dynamics governing the saturation or the dead-zone functions.

Acknowledgments. We thank Vincent Andrieu, Giacomo Casadei, and Sophie Tarbouriech for the fruitful discussions and suggestions that helped in the outcome of this work.

\section{ReFERENCES}

[1] J. Ahrens and H. Khalil, "High-gain observers in the presence of measurement noise: a switched-gain approach," Automatica, vol. 45, no. 4, pp. 936-943, 2009.

[2] A. Alessandri and M. Awawdeh, "Moving-horizon estimation with guaranteed robustness for discrete-time linear systems and measurements subject to outliers," Automatica, vol. 67, pp. 85-93, 2016.

[3] A. Alessandri, C. Cervellera, D. Macciò, and M. Sanguineti, "Optimization based on quasi-Monte Carlo sampling to design state estimators for nonlinear systems," Optimization, vol. 59, no. 7, pp. 963-984, 2010.
[4] A. Alessandri and A. Rossi, "Increasing-gain observers for nonlinear systems: stability and design," Automatica, vol. 57, no. 7, pp. 180-188, 2015.

[5] A. Alessandri and L. Zaccarian, "Results on stubborn Luenberger observers for linear time-invariant plants," in European Control Conf., 2015 , pp. 2925-2930.

[6] — "Stubborn state observers for linear time-invariant systems," Automatica, vol. 88, p. 1-9, 2018.

[7] V. Andrieu and L. Praly, "On the existence of a KazantzisKravaris/Luenberger observer," SIAM Journal on Control and Optimization, vol. 45, no. 2, pp. 432-456, 2006.

[8] M. Arcak and P. Kokotovic, "Nonlinear observer: a circle criterion design and robustness analysis," Automatica, vol. 37, no. 12, pp. 1923-1930, 2001.

[9] D. Astolfi, A. Alessandri, and L. Zaccarian, "Stubborn ISS redesign for nonlinear high-gain observers," in 20th IFAC World Congress, 2017, pp. 15992-15997.

[10] D. Astolfi, M. Jungers, and L. Zaccarian, "Output injection filtering redesign in high-gain observers," in European Control Conf., Jun. 2018, pp. 1957-1962.

[11] D. Astolfi and L. Marconi, "A high-gain nonlinear observer with limited gain power," IEEE Transactions on Automatic Control, vol. 60, no. 11, pp. 3059-3064, 2015.

[12] D. Astolfi, L. Marconi, L. Praly, and A. Teel, "Sensitivity to highfrequency measurement noise of nonlinear high-gain observers," IFACPapersOnLine, vol. 49, no. 18, pp. 862-866, 2016.

[13] _ , "Low-power peaking-free high-gain observers," Automatica, vol. 98, pp. 169-179, 2018.

[14] D. Astolfi and L. Praly, "Integral action in output feedback for multiinput multi-output nonlinear systems," IEEE Transactions on Automatic Control, vol. 62, no. 4, pp. 1559-1574, 2017.

[15] P. Bernard, L. Praly, and V. Andrieu, "Observers for a non-Lipschitz triangular form," Automatica, vol. 82, pp. 301-313, 2017.

[16] P. Bernard, L. Praly, V. Andrieu, and H. Hammouri, "On the triangular canonical form for uniformly observable controlled systems," Automatica, vol. 85, pp. 293-300, 2017.

[17] G. Besançon, G. Bornard, and H. Hammouri, "Observer synthesis for a class of nonlinear control systems," European Journal of Control, vol. 2, no. 3, pp. 176-192, 1996

[18] N. Boizot, E. Busvelle, and J.-P. Gauthier, "An adaptive high-gain observer for nonlinear systems," Automatica, vol. 46, no. 9, pp. 1483$1488,2010$.

[19] S. Bonnabel and J.-J. Slotine, "A contraction theory-based analysis of the stability of the deterministic extended Kalman filter," IEEE Transactions on Automatic Control, vol. 60, no. 2, pp. 565-569, 2015.

[20] K. K. Busawon and P. Kabore, "Disturbance attenuation using proportional integral observers," International Journal of control, vol. 74, no. 6 , pp. 618-627, 2001

[21] G. Casadei, D. Astolfi, A. Alessandri, and L. Zaccarian, "Synchronization in networks of identical nonlinear systems via dynamic dead zones," IEEE Control Systems Letters, vol. 3, no. 3, pp. 667-672, 2019.

[22] — - "Synchronization of interconnected linear systems via dynamic saturation redesign," IFAC-PapersOnLine, vol. 52, no. 16, pp. 622-627, 2019.

[23] M. Cocetti, S. Tarbouriech, and L. Zaccarian, "On dead-zone observers for linear plants," in Proc. American Control Conf., 2018, pp. 51385143.

[24] — , "High-gain dead-zone observers for linear and nonlinear plants," IEEE Control Systems Letters, vol. 3, no. 2, pp. 1-6, 2019.

[25] D. De Palma and G. Indiveri, "Output outlier robust state estimation," Int. J. of Adaptive Control and Signal Processing, vol. 31, no. 4, pp. 581-607, 2016

[26] H. Fang, M. Haile, and Y. Wang, "Robustifying the Kalman filter against measurement outliers: An innovation saturation mechanism," in 57th IEEE Conference on Decision and Control, 2018, pp. 6390-6395.

[27] J. Gauthier and I. Kupka, Deterministic Observation Theory and Applications. Cambridge University Press, 2001.

[28] M. Hamid, R. Postoyan, and J. Daafouz, "Local observers design for a class of neural mass models," in Proc. European Control Conf., 2015, pp. $1830-1835$.

[29] H. Hammouri and J. de Leon Morales, "Observer synthesis for stateaffine systems," in 29th IEEE Conference on Decision and Control, 1990, pp. 784-785.

[30] A. Isidori, Nonlinear control systems II. Springer Verlag, London, 1999.

[31] R. Kalman, "A new approach to linear filtering and prediction problems," Journal of Basic Engineering, vol. 82, no. 1, pp. 35-45, 1960.

[32] H. Khalil, Nonlinear Systems. Prentice Hall, 2002. 
[33] H. Khalil and L. Praly, "High-gain observers in nonlinear feedback control," Int. J. of Robust and Nonlinear Control, vol. 24, no. 6, pp. 993-1015, 2014.

[34] A. Krener, "The convergence of the extended Kalman filter," in Directions in mathematical systems theory and optimization. Springer, 2003, pp. 173-182.

[35] C. Lu, M. Wu, and Y. He, "Stubborn state estimation for delayed neural networks using saturating output errors," IEEE Transactions on Neural Networks and Learning Systems, 2019.

[36] D. Luenberger, "An introduction to observers," IEEE Transactions on Automatic Control, vol. 16, no. 6, pp. 596-602, March 1971.

[37] R. Rajamani, "Observers for Lipschitz nonlinear systems," IEEE Transactions on Automatic Control, vol. 43, no. 3, pp. 397-401, March 1998

[38] K. Reif, F. Sonnemann, and R. Unbehauen, "An EKF-based nonlinear observer with a prescribed degree of stability," Automatica, vol. 34, no. 9, pp. 1119-1123, 1998.

[39] H. Shim and D. Liberzon, "Nonlinear observers robust to measurement disturbances in an ISS sense," IEEE Transactions on Automatic Control, vol. 61, no. 1, pp. 48-61, 2016.

[40] H. Shim, J. H. Seo, and A. Teel, "Nonlinear observer design via passivation of error dynamics," Automatica, vol. 39, no. 5, pp. 885-892, 2003.

[41] E. Sontag, "Input to state stability: Basic concepts and results," in Nonlinear and optimal control theory. Springer, 2008, pp. 163-220.

[42] A. Teel, "Further variants of the Astolfi/Marconi high-gain observer," in Proc. American Control Conf., 2016, pp. 993-998.

[43] C. Tréangle, M. Farza, and M. M'Saad, "Filtered high gain observer for a class of uncertain nonlinear systems with sampled outputs," Automatica, vol. 101, pp. 197-206, 2019.

[44] W. Xu, E.-W. Bai, and M. Cho, "System identification in the presence of outliers and random noises: A compressed sensing approach," Automatica, vol. 50, no. 11, pp. 2905-2911, 2014.

[45] A. Zemouche and M. Boutayeb, "On LMI conditions to design observers for Lipschitz nonlinear systems," Automatica, vol. 49, pp. 585-591, 2013.

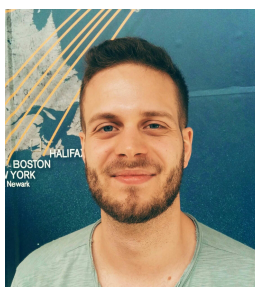

Daniele Astolfi received a joint Ph.D. degree in Control Theory from the University of Bologna, Italy, and from Mines ParisTech, France, in 2016. From 2016 to 2017, he has been a Research Assistant at CRAN, Nancy, France. Since 2018, he is a CNRS Researcher at LAGEPP, Lyon, France. His research interests include observer design, output regulation and synchronization for nonlinear and hybrid systems. He serves as an associate editor of the IFAC journal Automatica. He was a recipient of the 2016 Best Italian Ph.D. Thesis Award in Automatica given by SIDRA.

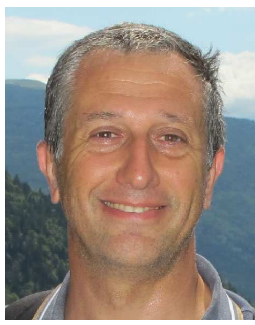

Angelo Alessandri (S'88-M'97-SM'02) received the "Laurea" degree and the Ph.D. degree in electronics engineering and computer science from the University of Genoa, Genoa, Italy, in 1992 and 1996, respectively. After a period as CNR research scientist, in 2005 he joined the University of Genoa, where he is currently an Associate Professor. His main research interests include moving-horizon estimation, state observers, and optimal control. $\mathrm{He}$ serves as an editor of the International Journal of Adaptive Control and Signal Processing and as an associate editor of the EUCA European Journal of Control and IFAC journal Automatica.

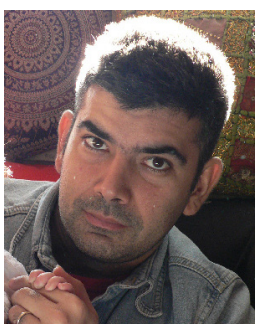

Luca Zaccarian (SM'09-F'16) received the Ph.D. degree in 2000 from the University of Roma Tor Vergata (Italy), where he has been Assistant and then Associate Professor from 2000 to 2011. Since 2011 he is Directeur de Recherche at the LAAS-CNRS, Toulouse (France) and since 2013 he holds a part-time Associate Professor position at the University of Trento, Italy. His main research interests include analysis and design of nonlinear/hybrid control systems, and control of mechatronic systems. He was a recipient of the 2001 O. Hugo Schuck Best Paper Award given by the American Automatic Control Council. 